TRANSACTIONS OF THE

AMERICAN MATHEMATICAL SOCIETY

Volume 363, Number 3, March 2011, Pages 1631-1664

S 0002-9947(2010)05227-4

Article electronically published on October 13, 2010

\title{
A REDUCTION OF THE TARGET OF THE JOHNSON HOMOMORPHISMS OF THE AUTOMORPHISM GROUP OF A FREE GROUP
}

\author{
TAKAO SATOH
}

\begin{abstract}
Let $F_{n}$ be a free group of rank $n$ and $F_{n}^{N}$ the quotient group of $F_{n}$ by a subgroup $\left[\Gamma_{n}(3), \Gamma_{n}(3)\right]\left[\left[\Gamma_{n}(2), \Gamma_{n}(2)\right], \Gamma_{n}(2)\right]$, where $\Gamma_{n}(k)$ denotes the $k$-th subgroup of the lower central series of the free group $F_{n}$. In this paper, we determine the group structure of the graded quotients of the lower central series of the group $F_{n}^{N}$ by using a generalized Chen's integration in free groups. Then we apply it to the study of the Johnson homomorphisms of the automorphism group of $F_{n}$. In particular, under taking a reduction of the target of the Johnson homomorphism induced from a quotient map $F_{n} \rightarrow F_{n}^{N}$, we see that there appear only two irreducible components, the Morita obstruction $S^{k} H_{\mathbf{Q}}$ and the Schur-Weyl module of type $H_{\mathbf{Q}}^{\left[k-2,1^{2}\right]}$, in the cokernel of the rational Johnson homomorphism $\tau_{k, \mathbf{Q}}^{\prime}=\tau_{k}^{\prime} \otimes \operatorname{id}_{\mathbf{Q}}$ for $k \geq 5$ and $n \geq k+2$.
\end{abstract}

\section{INTRODUCTION}

Let $F_{n}$ be a free group of rank $n \geq 2$, and let Aut $F_{n}$ be the automorphism group of $F_{n}$. Let $\rho$ : Aut $F_{n} \rightarrow$ Aut $H$ denote the natural homomorphism induced from the abelianization $F_{n} \rightarrow H$ of $F_{n}$. The kernel of $\rho$ is called the IA-automorphism group of $F_{n}$, denoted by $\mathrm{IA}_{n}$. The group $\mathrm{IA}_{n}$ reflects much of the richness and complexity of the structure of Aut $F_{n}$ and plays important roles on various studies of Aut $F_{n}$.

Although the study of the IA-automorphism group has a long history, the combinatorial group structure of $\mathrm{IA}_{n}$ is still quite complicated. In 1935, Magnus [14] obtained finitely many generators of $\mathrm{IA}_{n}$. Nielsen [21] showed that $\mathrm{IA}_{2}$ coincides with the inner automorphism group of $F_{2}$; hence, it is isomorphic to $F_{2}$. In general, however, any presentation for $\mathrm{IA}_{n}$ is not known. Krstić and McCool [13] showed that $\mathrm{IA}_{3}$ is not finitely presentable. For $n \geq 4$, it is also not known whether $\mathrm{IA}_{n}$ is finitely presentable or not.

The purpose of our research is to clarify the group structure of $\mathrm{IA}_{n}$. In particular, we are interested in determining the graded quotients of the Johnson filtration of Aut $F_{n}$. The Johnson filtration is a descending central series

$$
\mathrm{IA}_{n}=\mathcal{A}_{n}(1) \supset \mathcal{A}_{n}(2) \supset \cdots
$$

Received by the editors June 10, 2009 and, in revised form, October 15, 2009.

2000 Mathematics Subject Classification. Primary 20F28; Secondary $20 \mathrm{~F} 12$.

Key words and phrases. Automorphism group of a free group, IA-automorphism group, Johnson homomorphisms, Chen's integration on free groups. 
consisting of normal subgroups of Aut $F_{n}$. Then a homomorphism

$$
\tilde{\tau}_{k}: \mathcal{A}_{n}(k) \rightarrow H^{*} \otimes_{\mathbf{z}} \mathcal{L}_{n}(k+1)
$$

is defined by $\tilde{\tau}_{k}(\sigma)=\left(x \mapsto x^{-1} x^{\sigma}\right)$ for each $k \geq 1$. The map $\tilde{\tau}_{k}$ induces a homomorphism

$$
\tau_{k}: \operatorname{gr}^{k}\left(\mathcal{A}_{n}\right) \rightarrow H^{*} \otimes \mathbf{z} \mathcal{L}_{n}(k+1)
$$

from the $k$-th graded quotient of the Johnson filtration. Both $\tilde{\tau}_{k}$ and $\tau_{k}$ are called the $k$-th Johnson homomorphisms of the automorphism group of a free group. In particular, $\tau_{k}$ is a $\operatorname{GL}(n, \mathbf{Z})$-equivariant injective homomorphism. (For the details, see Subsection 2.5.) The study of the Johnson homomorphisms was originally begun in 1980 by D. Johnson [10] who determined the abelianization of the Torelli subgroup of a mapping class group of a surface in [11. Recently, the study of the Johnson filtration and the Johnson homomorphisms of Aut $F_{n}$ achieved good progress through the work of many authors, for example, [7, 12, [18, [19, [20, 24] and 26].

Through the images of the Johnson homomorphisms, we can study $\mathrm{IA}_{n}$ using infinitely many pieces of a free abelian group of finite rank. They are regarded as one-by-one approximations of $\mathrm{IA}_{n}$, and to clarify the structure of them plays an important role in various studies of $\mathrm{IA}_{n}$. In this paper, we are interested in determining the $\mathrm{GL}(n, \mathbf{Z})$-module structure of the cokernel of the rational Johnson homomorphisms $\tau_{k, \mathbf{Q}}=\tau_{k} \otimes \operatorname{id}_{\mathbf{Q}}$. Now, for $1 \leq k \leq 3$, the cokernel of $\tau_{k, \mathbf{Q}}$ is completely determined. (See 1], 24 and [26 for $k=1,2$ and 3, respectively.) Recently, Morita [19, 20] showed that for each $k \geq 2$, there appears the symmetric tensor product $S^{k} H_{\mathbf{Q}}$ of $H_{\mathbf{Q}}:=H_{\mathbf{Z}} \otimes \mathbf{Q}$ in the irreducible decomposition of $\operatorname{Coker}\left(\tau_{k, \mathbf{Q}}\right)$ using trace maps. The modules $S^{k} H_{\mathbf{Q}}$ are the first obstructions for the surjectivity of the Johnson homomorphisms, discovered by Morita. We call them the Morita obstructions. In general, however, it is quite a hard problem to determine $\operatorname{Coker}\left(\tau_{k, \mathbf{Q}}\right)$. Even its $\mathbf{Q}$-dimension is not calculated for $k \geq 4$. One reason for the difficulty is that we cannot study the image of the Johnson homomorphisms directly since there is little information for generators of the graded quotients $\operatorname{gr}^{k}\left(\mathcal{A}_{n}\right)$.

To avoid this difficulty, we consider the lower central series $\mathcal{A}_{n}^{\prime}(1)=\mathrm{IA}_{n}, \mathcal{A}_{n}^{\prime}(2)$, $\ldots$ of $\mathrm{IA}_{n}$. Since the Johnson filtration is central, $\mathcal{A}_{n}^{\prime}(k) \subset \mathcal{A}_{n}(k)$ for $k \geq 1$. It was conjectured that $\mathcal{A}_{n}^{\prime}(k)=\mathcal{A}_{n}(k)$ for each $k \geq 1$ by Andreadakis, who showed that $\mathcal{A}_{2}^{\prime}(k)=\mathcal{A}_{2}(k)$ for each $k \geq 1$ and $\mathcal{A}_{3}^{\prime}(3)=\mathcal{A}_{3}(3)$ in [1. Now, we have $\mathcal{A}_{n}^{\prime}(2)=\mathcal{A}_{n}(2)$ due to Cohen-Pakianathan [3, 4], Farb [5] and Kawazumi [12. (See (3) below.) Furthermore, $\mathcal{A}_{n}^{\prime}(3)$ has at most a finite index in $\mathcal{A}_{n}(3)$ due to Pettet [24. It is, however, also difficult to determine whether $\mathcal{A}_{n}^{\prime}(k)$ coincides with $\mathcal{A}_{n}(k)$ or not.

For each $k \geq 1, \operatorname{set} \operatorname{gr}^{k}\left(\mathcal{A}_{n}^{\prime}\right):=\mathcal{A}_{n}^{\prime}(k) / \mathcal{A}_{n}^{\prime}(k+1)$. We can also define the Johnson homomorphisms

$$
\tau_{k}^{\prime}: \operatorname{gr}^{k}\left(\mathcal{A}_{n}^{\prime}\right) \rightarrow H^{*} \otimes_{\mathbf{z}} \mathcal{L}_{n}(k+1)
$$

by an argument similar to that in the definition of $\tau_{k}$. In general, we can consider $\operatorname{Coker}\left(\tau_{k, \mathbf{Q}}\right)$ as a $\mathrm{GL}(n, \mathbf{Z})$-equivariant submodule in $\operatorname{Coker}\left(\tau_{k, \mathbf{Q}}^{\prime}\right)$. Namely, by studying the structure of $\operatorname{Coker}\left(\tau_{k, \mathbf{Q}}^{\prime}\right)$, we obtain an upper bound on $\operatorname{Coker}\left(\tau_{k, \mathbf{Q}}\right)$. Furthermore the most important thing is that since $\mathrm{IA}_{n}$ is finitely generated by the Magnus generators, each $\operatorname{gr}^{k}\left(\mathcal{A}_{n}^{\prime}\right)$ is also finitely generated by commutators 
of weight $k$ among them. Therefore, it is more accessible to study the cokernel of $\tau_{k}^{\prime}$ than that of $\tau_{k}$. Now, it is known that $\operatorname{Coker}\left(\tau_{k, \mathbf{Q}}^{\prime}\right)=\operatorname{Coker}\left(\tau_{k, \mathbf{Q}}\right)$ for $1 \leq k \leq 3$. In our previous paper [28, we determined the $\mathrm{GL}(n, \mathbf{Z})$-module structure of $\operatorname{Coker}\left(\tau_{4, \mathbf{Q}}^{\prime}\right)$ for $n \geq 6$. However, to determine the structure of $\operatorname{Coker}\left(\tau_{k, \mathbf{Q}}^{\prime}\right)$ is still complicated in general.

One of the main purposes of the paper is to consider a reduction of the target of the Johnson homomorphism $\tau_{k}^{\prime}$. More precisely, let $F_{n}^{N}$ be the quotient group of $F_{n}$ by the subgroup $\left[\Gamma_{n}(3), \Gamma_{n}(3)\right]\left[\left[\Gamma(2), \Gamma_{n}(2)\right], \Gamma_{n}(2)\right]$. If we let $\Gamma_{n}^{N}(k)$ be the lower central series of $F_{n}^{N}$ and set $\mathcal{L}_{n}^{N}(k):=\Gamma_{n}^{N}(k) / \Gamma_{n}^{N}(k+1)$, we have a natural map

$$
H^{*} \otimes_{\mathbf{z}} \mathcal{L}_{n}(k+1) \rightarrow H^{*} \otimes_{\mathbf{z}} \mathcal{L}_{n}^{N}(k+1)
$$

In this paper, we consider the composition

$$
\tau_{k, N}^{\prime}: \operatorname{gr}^{k}\left(\mathcal{A}_{n}^{\prime}\right) \rightarrow H^{*} \otimes_{\mathbf{z}} \mathcal{L}_{n}^{N}(k+1)
$$

of $\tau_{k}^{\prime}$ and the natural projection above. The map $\tau_{k, N}^{\prime}$ is a $\operatorname{GL}(n, \mathbf{Z})$-equivariant homomorphism. Then we show

Theorem 1 (= Theorem 5.3). For $k \geq 5$ and $n \geq k+2$,

$$
\operatorname{Coker}\left(\left(\tau_{k, N}^{\prime}\right)_{\mathbf{Q}}\right)=S^{k} H_{\mathbf{Q}} \oplus H_{\mathbf{Q}}^{\left[k-2,1^{2}\right]},
$$

where $H_{\mathbf{Q}}^{\left[k-2,1^{2}\right]}$ denotes the Schur-Weyl module of $H_{\mathbf{Q}}$ corresponding to the partition $\left[k-2,1^{2}\right]$ of $k$.

This shows that $H_{\mathbf{Q}}^{\left[k-2,1^{2}\right]}$ also appears in the irreducible decomposition of $\operatorname{Coker}\left(\tau_{k, \mathbf{Q}}^{\prime}\right)$ for $n \geq k+2$. This work is an analogue and a certain extension of our previous work 27] in which we were concerned with the Johnson homomorphisms of the automorphism group of a free metabelian group. In particular, we showed that there appears only the Morita obstruction in the cokernel of it.

The reason why we consider the quotient group $F_{n}^{N}$ is that the structure of the graded quotients $\mathcal{L}_{n}^{N}(k)$ of the lower central series of $F_{n}^{N}$ is easier to handle than that of the other quotient group of $F_{n}$, for example $F_{n} /\left[\Gamma_{n}(3), \Gamma_{n}(3)\right]$, except for a free metabelian group. In general, although to give an irreducible decomposition of $\operatorname{Coker}\left(\tau_{k, \mathbf{Q}}^{\prime}\right)$ is difficult, considering such a reduction of the target of the Johnson homomorphism $\tau_{k}^{\prime}$, we can find a new obstruction for the surjectivity of $\tau_{k, \mathbf{Q}}^{\prime}$.

Before showing Theorem 1, we have to determine the group structure of each $\mathcal{L}_{n}^{N}(k)$ for $k \geq 6$. The other purpose of the paper is to show

Theorem 2 (= Theorem 4.1 and Corollary 4.1). For $n \geq 6$, each of $\mathcal{L}_{n}^{N}(k)$ is a free abelian group with

$$
\operatorname{rank}_{\mathbf{Z}}\left(\mathcal{L}_{n}^{N}(k)\right)=(k-1)\left(\begin{array}{c}
k+n-2 \\
k
\end{array}\right)+\frac{1}{2} n(n-1)(k-3)\left(\begin{array}{c}
n+k-4 \\
k-2
\end{array}\right) .
$$

In general, it is easy to show that each $\mathcal{L}_{n}^{N}(k)$ is a finitely generated abelian group. Hence the difficult part is to show that $\mathcal{L}_{n}^{N}(k)$ is free and to determine its rank. To do this, we introduce a certain integration

$$
I_{j}\left(f, w ; a_{1}, \ldots, a_{n}\right):=\int_{l_{w}\left(a_{1}, \ldots, a_{n}\right)} f(t) d t_{j}
$$


in Section 3 This is a generalization of Chen's integration in free groups introduced by K. T. Chen who determined the group structure of the graded quotients of the lower central series of a free metabelian group in 2 .

This paper consists of six sections. In Section 2, we recall the associated Lie algebra of a group, the IA-automorphism group and the Johnson homomorphisms. In Section 3, we introduce a generalization of Chen's integration in free groups, and study some properties. In Section 4 , we determine the group structure of the graded quotient $\mathcal{L}_{n}^{N}(k)$ of the lower central series of $F_{n}^{N}$. Finally, in Section 5 , we determine the cokernel of $\left(\tau_{k, N}^{\prime}\right)_{\mathbf{Q}}$.

\section{Preliminaries}

In this section, we recall the definition and some properties of the associated Lie algebra of a group $G$, the IA-automorphism group of a free group and the Johnson homomorphisms of Aut $F_{n}$.

2.1. Notation and conventions. Throughout the paper, we use the following notation and conventions. Let $G$ be a group and $N$ a normal subgroup of $G$.

- The abelianization of $G$ is denoted by $G^{\text {ab }}$.

- The group Aut $G$ of $G$ acts on $G$ from the right. For any $\sigma \in$ Aut $G$ and $x \in G$, the action of $\sigma$ on $x$ is denoted by $x^{\sigma}$.

- For an element $g \in G$, we also denote the coset class of $g$ by $g \in G / N$ if there is no confusion.

- For any $\mathbf{Z}$-module $M$, we denote by $M \otimes_{\mathbf{Z}} \mathbf{Q}$ the symbol obtained by attaching a subscript $\mathbf{Q}$ to $M$, such as $M_{\mathbf{Q}}$ or $M^{\mathbf{Q}}$. Similarly, for any $\mathbf{Z}$ linear map $f: A \rightarrow B$, the induced $\mathbf{Q}$-linear map $A_{\mathbf{Q}} \rightarrow B_{\mathbf{Q}}$ is denoted by $f_{\mathbf{Q}}$ or $f^{\mathbf{Q}}$.

- For each $k \geq 1$, and any partition $\lambda$ of $k$, we denote by $H^{\lambda}$ the SchurWeyl module of $H$ corresponding to the partition $\lambda$ of $k$. For example, the modules $H^{[k]}$ and $H^{\left[1^{k}\right]}$ are the symmetric product $S^{k} H$ and the exterior product $\Lambda^{k} H$, respectively. (For details, see [6].)

- For elements $x$ and $y$ of $G$, the commutator bracket $[x, y]$ of $x$ and $y$ is defined to be $[x, y]:=x y x^{-1} y^{-1}$.

2.2. Associated Lie algebra of a group. Let $G$ be a group, and let $\Gamma_{G}(k)$ be the $k$-th term of the lower central series of $G$ defined by

$$
\Gamma_{G}(1):=G, \quad \Gamma_{G}(k):=\left[\Gamma_{G}(k-1), G\right], \quad k \geq 2 .
$$

For each $k \geq 1$, set $\mathcal{L}_{G}(k):=\Gamma_{G}(k) / \Gamma_{G}(k+1)$ and

$$
\mathcal{L}_{G}:=\bigoplus_{k \geq 1} \mathcal{L}_{G}(k)
$$

Then $\mathcal{L}_{G}$ has a graded Lie algebra structure induced from the commutator bracket on $G$. We call $\mathcal{L}_{G}$ the associated Lie algebra of a group $G$. Clearly, the correspondence from $G$ to $\mathcal{L}_{G}$ is a covariant functor from the category of groups to that of graded Lie algebras. In particular, if $f: G_{1} \rightarrow G_{2}$ is a surjective group homomorphism, the induced homomorphism $f_{*}: \mathcal{L}_{G_{1}} \rightarrow \mathcal{L}_{G_{2}}$ is also surjective.

For any $g_{1}, \ldots, g_{k} \in G$, a commutator of weight $k$ among the components $g_{1}, \ldots, g_{k}$ of the type

$$
\left[\left[\cdots\left[\left[g_{1}, g_{2}\right], g_{3}\right], \cdots\right], g_{k}\right]
$$


with all of its brackets to the left of all the elements occurring is called a simple $k$-fold commutator, denoted by $\left[g_{1}, g_{2}, \ldots, g_{k}\right]$. In general, if $G$ is generated by $g_{1}, \ldots, g_{n}$, then for each $k \geq 1, \mathcal{L}_{G}(k)$ is generated by (the coset classes of) the simple $k$-fold commutators

$$
\left[g_{i_{1}}, g_{i_{2}}, \ldots, g_{i_{k}}\right], \quad i_{j} \in\{1, \ldots, n\} .
$$

For details, see [15] for example.

Next we consider the case where $G$ is a free group $F_{n}$ on $x_{1}, \ldots, x_{n}$. For simplicity, we write $\Gamma_{n}(k), \mathcal{L}_{n}(k)$ and $\mathcal{L}_{n}$ for $\Gamma_{G}(k), \mathcal{L}_{G}(k)$ and $\mathcal{L}_{G}$, respectively. The associated Lie algebra $\mathcal{L}_{n}$ is called the free Lie algebra generated by $H$. (See [25] for basic materials concerning the free Lie algebra.) It is classically well known due to Witt [29] that for each $k \geq 1$, the graded quotient $\mathcal{L}_{n}(k)$ is a $\operatorname{GL}(n, \mathbf{Z})$-equivariant free abelian group of rank

$$
r_{n}(k):=\frac{1}{k} \sum_{d \mid k} \mu(d) n^{\frac{k}{d}},
$$

where $\mu$ is the Möbius function.

Now, we denote by $F_{n}^{M}$ the quotient group of $F_{n}$ by a subgroup $\left[\Gamma_{n}(2), \Gamma_{n}(2)\right]$. The group $F_{n}^{M}$ is called a free metabelian group of rank $n$. For simplicity, we write $\Gamma_{n}^{M}(k), \mathcal{L}_{n}^{M}(k)$ and $\mathcal{L}_{n}^{M}$ for $\Gamma_{F_{n}^{M}}(k), \mathcal{L}_{F_{n}^{M}}(k)$ and $\mathcal{L}_{F_{n}^{M}}$, respectively. The associated Lie algebra $\mathcal{L}_{n}^{M}$ is called the free metabelian algebra generated by $H$, or the Chen Lie algebra. By a remarkable work by Chen [2], it is known that for each $k \geq 1$ the graded quotient $\mathcal{L}_{n}^{M}(k)$ is a $\mathrm{GL}(n, \mathbf{Z})$-equivariant free abelian group of rank

$$
r_{n}^{M}(k):=(k-1)\left(\begin{array}{c}
n+k-2 \\
k
\end{array}\right)
$$

with basis

$$
\left\{\left[x_{i_{1}}, x_{i_{2}}, \ldots, x_{i_{k}}\right] \mid i_{1}>i_{2} \leq i_{3} \leq \cdots \leq i_{k}\right\} .
$$

Let $F_{n}^{N}$ be the quotient group of $F_{n}$ by the subgroup $\left[\Gamma_{n}(3), \Gamma_{n}(3)\right]\left[\left[\Gamma(2), \Gamma_{n}(2)\right]\right.$, $\left.\Gamma_{n}(2)\right]$. For simplicity, we write $\Gamma_{n}^{N}(k), \mathcal{L}_{n}^{N}(k)$ and $\mathcal{L}_{n}^{N}$ for $\Gamma_{F_{n}^{N}}(k), \mathcal{L}_{F_{n}^{N}}(k)$ and $\mathcal{L}_{F_{n}^{N}}$, respectively. In Section 4 , we determine the rank of $\mathcal{L}_{n}^{N}(k)$ for each $k \geq 1$.

2.3. Hall basis. Here, we recall the Hall basis of $\mathcal{L}_{n}(k)$ for each $k \geq 1$. In [8], P. Hall introduced basic commutators of $F_{n}$ and showed that those of weight $k$ form a basis of $\mathcal{L}_{n}(k)$. Now, it is called the Hall basis of $\mathcal{L}_{n}(k)$. (For details for the basic commutators, see [9] and 25] for example.) In this paper, we consider a fixed sequence of basic commutators of $F_{n}$ beginning with

$$
x_{1}<x_{2}<\cdots<x_{n}<\left[x_{2}, x_{1}\right]<\left[x_{3}, x_{1}\right]<\left[x_{3}, x_{2}\right]<\cdots<\left[x_{n}, x_{n-1}\right]<\cdots,
$$

where the ordering among $\left[x_{i}, x_{j}\right]$ is defined by the lexicographic ordering.

Let $c_{l, 1}<\cdots<c_{l, m_{l}}$ be the basic commutators of weight $l$. If $w$ is a product of basic commutators of weight $\geq l$, and if we apply the Hall's correcting process to $w$, then for each $k \geq l, w$ is rewritten as a form

$$
w=c_{l, 1}^{e_{l, 1}} \cdots c_{l, m_{l}}^{e_{l, m_{l}}} \cdots c_{k, 1}^{e_{k, 1}} \cdots c_{k, m_{k}}^{e_{k, m_{k}}} w^{\prime},
$$

where $w^{\prime}$ is a product of commutators $\left[u_{1}, u_{2}, \ldots, u_{r}\right]$ in $\Gamma_{n}(k+1)$ and each element $u_{i}$ of the component is in $\Gamma_{n}(l)$. (For details for the correcting process, see [9].) 
In particular, from the above we see that for each $k \geq 1$, any element $w \in F_{n}$ is uniquely written as a form

$$
w \equiv c_{1,1}^{e_{1,1}} \cdots c_{1, n}^{e_{1, n}} \cdots c_{k, 1}^{e_{k, 1}} \cdots c_{k, m_{k}}^{e_{k, m_{k}}} \quad\left(\bmod \Gamma_{n}(k+1)\right)
$$

for some $e_{i, m_{i}} \in \mathbf{Z}$. We call it the $\bmod -\Gamma_{n}(k+1)$ normal form of $w$.

For any $k \geq 2$, the basic commutators which do not belong to $\left[\Gamma_{n}(2), \Gamma_{n}(2)\right]$ are $\left[x_{i_{1}}, x_{i_{2}}, \ldots, x_{i_{k}}\right]$ for $i_{1}>i_{2} \leq i_{3} \leq \cdots \leq i_{k}$.

2.4. IA-automorphism group. Let $\rho:$ Aut $F_{n} \rightarrow$ Aut $H$ be the natural homomorphism induced from the abelianization $F_{n} \rightarrow H$ of $F_{n}$. In this paper we identify Aut $H$ with the general linear group $\operatorname{GL}(n, \mathbf{Z})$ by fixing the basis of $H$ as a free abelian group induced from the basis $x_{1}, \ldots, x_{n}$ of $F_{n}$. The kernel IA $\mathrm{I}_{n}$ of $\rho$ is called the IA-automorphism group of $F_{n}$. Magnus [14] showed that for any $n \geq 3, \mathrm{IA}_{n}$ is finitely generated by automorphisms

$$
K_{i j}:\left\{\begin{array}{ll}
x_{i} & \mapsto x_{j}{ }^{-1} x_{i} x_{j}, \\
x_{t} & \mapsto x_{t}
\end{array} \quad(t \neq i)\right.
$$

for distinct $i, j \in\{1,2, \ldots, n\}$ and

$$
K_{i j l}:\left\{\begin{array}{lll}
x_{i} & \mapsto x_{i} x_{j} x_{l} x_{j}{ }^{-1} x_{l}{ }^{-1} \\
x_{t} & \mapsto x_{t} & (t \neq i)
\end{array}\right.
$$

for distinct $i, j, l \in\{1,2, \ldots, n\}$ such that $j>l$.

Recently, Cohen-Pakianathan [3, 4, Farb [5] and Kawazumi [12] independently showed that the abelianization of $\mathrm{IA}_{n}$ is a free abelian group, and the Magnus generators above induce a basis of it. More precisely, they showed

$$
\mathrm{IA}_{n}^{\mathrm{ab}} \cong H^{*} \otimes \mathbf{z} \Lambda^{2} H
$$

as a $\operatorname{GL}(n, \mathbf{Z})$-module where $H^{*}:=\operatorname{Hom}_{\mathbf{Z}}(H, \mathbf{Z})$ denotes the dual group of $H$.

2.5. Johnson homomorphisms. In this subsection, we recall the Johnson homomorphisms of the automorphism group of a free group. To begin with, we recall a descending filtration of Aut $F_{n}$ called the Johnson filtration. For $k \geq 0$, the action of Aut $F_{n}$ on each nilpotent quotient $F_{n} / \Gamma_{n}(k+1)$ of $F_{n}$ induces a homomorphism

$$
\text { Aut } F_{n} \rightarrow \operatorname{Aut}\left(F_{n} / \Gamma_{n}(k+1)\right) \text {. }
$$

We denote the kernel of it by $\mathcal{A}_{n}(k)$. Then the groups $\mathcal{A}_{n}(k)$ define a descending central filtration

$$
\text { Aut } F_{n}=\mathcal{A}_{n}(0) \supset \mathcal{A}_{n}(1) \supset \mathcal{A}_{n}(2) \supset \cdots
$$

of Aut $F_{n}$, with $\mathcal{A}_{n}(1)=\mathrm{IA}_{n}$. (See [1] for details.) It is called the Johnson filtration of Aut $F_{n}$. For each $k \geq 1$, the group Aut $F_{n}$ acts on $\mathcal{A}_{n}(k)$ by conjugation, and it naturally induces an action of $\operatorname{GL}(n, \mathbf{Z})$ on $\operatorname{gr}^{k}\left(\mathcal{A}_{n}\right):=\mathcal{A}_{n}(k) / \mathcal{A}_{n}(k+1)$. The graded sum $\operatorname{gr}\left(\mathcal{A}_{n}\right):=\bigoplus_{k \geq 1} \operatorname{gr}^{k}\left(\mathcal{A}_{n}\right)$ has a graded Lie algebra structure induced from the commutator bracket on $\mathrm{IA}_{n}$.

In order to study the $\mathrm{GL}(n, \mathbf{Z})$-module structure of $\operatorname{gr}^{k}\left(\mathcal{A}_{n}\right)$ for each $k \geq 1$, we consider the Johnson homomorphisms of Aut $F_{n}$ as follows. For each $k \geq 1$, define a homomorphism $\tilde{\tau}_{k}: \mathcal{A}_{n}(k) \rightarrow \operatorname{Hom}_{\mathbf{Z}}\left(H, \mathcal{L}_{n}(k+1)\right)$ by

$$
\sigma \mapsto\left(x \mapsto x^{-1} x^{\sigma}\right), \quad x \in H .
$$


Then the kernel of $\tilde{\tau}_{k}$ is just $\mathcal{A}_{n}(k+1)$. Hence it induces an injective homomorphism

$$
\tau_{k}: \operatorname{gr}^{k}\left(\mathcal{A}_{n}\right) \hookrightarrow \operatorname{Hom}_{\mathbf{Z}}\left(H, \mathcal{L}_{n}(k+1)\right)=H^{*} \otimes_{\mathbf{z}} \mathcal{L}_{n}(k+1) .
$$

The homomorphisms $\tilde{\tau}_{k}$ and $\tau_{k}$ are called the $k$-th Johnson homomorphisms of Aut $F_{n}$. It is easily seen that each $\tau_{k}$ is a $\operatorname{GL}(n, \mathbf{Z})$-equivariant injective homomorphism. For the Magnus generators of $\mathrm{IA}_{n}$, their images by $\tau_{1}$ are given by

$$
\tau_{1}\left(K_{i j}\right)=x_{i}^{*} \otimes\left[x_{i}, x_{j}\right], \quad \tau_{1}\left(K_{i j l}\right)=x_{i}^{*} \otimes\left[x_{j}, x_{l}\right] .
$$

Furthermore, we remark that $\tau_{1}\left(=\tau_{1}^{\prime}\right)$ is just the abelianization of $\mathrm{IA}_{n}$. (See 3, 4, 5, 12.)

Let $\operatorname{Der}\left(\mathcal{L}_{n}\right)$ be the graded Lie algebra of derivations of $\mathcal{L}_{n}$. The degree $k$ part of $\operatorname{Der}\left(\mathcal{L}_{n}\right)$ is considered as $H^{*} \otimes_{\mathbf{z}} \mathcal{L}_{n}(k+1)$, and we identify them in this paper. Then the sum of the Johnson homomorphisms

$$
\tau:=\bigoplus_{k \geq 1} \tau_{k}: \operatorname{gr}\left(\mathcal{A}_{n}\right) \rightarrow \operatorname{Der}\left(\mathcal{L}_{n}\right)
$$

is a graded Lie algebra homomorphism. In fact, if we denote by $\partial \xi$ the element of $\operatorname{Der}\left(\mathcal{L}_{n}\right)$ corresponding to an element $\xi \in H^{*} \otimes_{\mathbf{z}} \mathcal{L}_{n}$, and write the action of $\partial \xi$ on $X \in \mathcal{L}_{n}$ as $X^{\partial \xi}$, then we have

$$
\tau_{k+l}\left(\left[\sigma, \sigma^{\prime}\right]\right)=\tau_{k}(\sigma)^{\partial \tau_{l}\left(\sigma^{\prime}\right)}-\tau_{l}\left(\sigma^{\prime}\right)^{\partial \tau_{k}(\sigma)}
$$

for any $\sigma \in \mathcal{A}_{n}(k)$ and $\sigma^{\prime} \in \mathcal{A}_{n}(l)$. This formula is very useful for calculating the image of the Johnson homomorphism inductively.

For $1 \leq k \leq 4$, the irreducible decomposition of the cokernel of the rational Johnson homomorphism $\tau_{k, \mathbf{Q}}$ and the rank of $\operatorname{gr}^{k}\left(\mathcal{A}_{n}\right)$ are obtained as follows:

\begin{tabular}{l|l|l|l}
\hline$k$ & $\operatorname{Coker}\left(\tau_{k, \mathbf{Q}}\right)$ & $\operatorname{rank}_{\mathbf{Z}}\left(\operatorname{gr}^{k}\left(\mathcal{A}_{n}\right)\right)$ & \\
\hline 1 & 0 & $n^{2}(n-1) / 2$ & Andreadakis [1] \\
\hline 2 & $S^{2} H_{\mathbf{Q}}$ & $n(n+1)\left(2 n^{2}-2 n-3\right) / 6$ & Pettet [24] \\
\hline 3 & $S^{3} H_{\mathbf{Q}} \oplus \Lambda^{3} H_{\mathbf{Q}}$ & $n\left(3 n^{4}-7 n^{2}-8\right) / 12$ & Satoh [26] \\
\hline
\end{tabular}

In general, however, to determine the structure of the image and the cokernel of $\tau_{k}$ is quite difficult.

Let $\mathcal{A}_{n}^{\prime}(k)$ be the lower central series of $\mathrm{IA}_{n}$ with $\mathcal{A}_{n}^{\prime}(1)=\mathrm{IA}_{n}$. Since the Johnson filtration is central, $\mathcal{A}_{n}^{\prime}(k) \subset \mathcal{A}_{n}(k)$ for each $k \geq 1$. $\operatorname{Set} \operatorname{gr}^{k}\left(\mathcal{A}_{n}^{\prime}\right):=$ $\mathcal{A}_{n}^{\prime}(k) / \mathcal{A}_{n}^{\prime}(k+1)$ and $\operatorname{gr}\left(\mathcal{A}_{n}^{\prime}\right):=\bigoplus_{k>1} \operatorname{gr}^{k}\left(\mathcal{A}_{n}^{\prime}\right)$. Then $\operatorname{gr}\left(\mathcal{A}_{n}^{\prime}\right)$ is also a graded Lie algebra induced from the commutator bracket on $\operatorname{IA}_{n}$, and $\operatorname{GL}(n, \mathbf{Z})$ naturally acts on each of $\operatorname{gr}^{k}\left(\mathcal{A}_{n}^{\prime}\right)$. Moreover, since $\mathrm{IA}_{n}$ is finitely generated by the Magnus generators $K_{i j}$ and $K_{i j l}$, each $\operatorname{gr}^{k}\left(\mathcal{A}_{n}^{\prime}\right)$ is also finitely generated by the simple $k$-fold commutators among the components $K_{i j}$ and $K_{i j l}$.

A restriction of $\tilde{\tau}_{k}$ to $\mathcal{A}_{n}^{\prime}(k)$ induces a $\operatorname{GL}(n, \mathbf{Z})$-equivariant homomorphism

$$
\tau_{k}^{\prime}: \operatorname{gr}^{k}\left(\mathcal{A}_{n}^{\prime}\right) \rightarrow H^{*} \otimes_{\mathbf{Z}} \mathcal{L}_{n}(k+1),
$$

and the sum

$$
\tau^{\prime}:=\bigoplus_{k \geq 1} \tau_{k}^{\prime}: \operatorname{gr}\left(\mathcal{A}_{n}^{\prime}\right) \rightarrow \operatorname{Der}\left(\mathcal{L}_{n}\right)
$$


is also a graded Lie algebra homomorphism. Furthermore, we have

$$
\tau_{k+l}^{\prime}\left(\left[\sigma, \sigma^{\prime}\right]\right)=\tau_{k}^{\prime}(\sigma)^{\partial \tau_{l}\left(\sigma^{\prime}\right)}-\tau_{l}^{\prime}\left(\sigma^{\prime}\right)^{\partial \tau_{k}(\sigma)}
$$

for any $\sigma \in \mathcal{A}_{n}^{\prime}(k)$ and $\sigma^{\prime} \in \mathcal{A}_{n}^{\prime}(l)$. Using this formula recursively, we can easily compute $\tau_{k}^{\prime}(\sigma)$ for any $\sigma \in \mathcal{A}_{n}^{\prime}(k)$ from (4). We should remark that, in general, it is not known whether $\tau_{k}^{\prime}$ is injective or not. In this paper, we study the cokernel of the rational Johnson homomorphism $\tau_{k, \mathbf{Q}}^{\prime}=\tau_{k}^{\prime} \otimes \operatorname{id}_{\mathbf{Q}}$.

\section{A generalization of Chen's integration in free groups}

In this section, we introduce a generalization of Chen's integration in free groups which is used to determine the structure of the graded quotients $\mathcal{L}_{n}^{N}(k)$ in Section 4.

Given the free group $F_{n}$ generated by $x_{1}, \ldots, x_{n}$, denote by $\mathbf{E}$ the vector space over the real field $\mathbf{R}$ with basis $x_{1}, \ldots, x_{n}$ and $\left[x_{i}, x_{j}\right]$ for $1 \leq j<i \leq n$. A Euclidean metric is introduced into $\mathbf{E}$ by taking $x_{1}, \ldots, x_{n}$ and $\left[x_{i}, x_{j}\right]$ as an orthonormal basis. Then $\mathbf{E}$ is a Euclidean $n(n+1) / 2$-space. The orthonormal basis induces a Cartesian coordinate system in $\mathbf{E}$. We call the coordinates corresponding to $x_{i}$ and $\left[x_{i}, x_{j}\right]$ the $t_{i}$-coordinates and the $t_{i, j}$-coordinates, respectively.

Let $\Omega_{n}$ be the set of words among the letters $x_{1}, \ldots, x_{n}$. A quotient set of $\Omega_{n}$ by an equivalence relation induced from $x_{i}^{e} x_{i}^{-e}=1$ for $e= \pm 1$ forms the free group $F_{n}$. For any word $w=x_{i_{1}}^{e_{1}} x_{i_{2}}^{e_{2}} \cdots x_{i_{m}}^{e_{m}}$ with $e_{k}= \pm 1$, and any integers $a_{1}, \ldots, a_{n} \in \mathbf{Z}$, we define points $P_{s} \in \mathbf{E}$ for $0 \leq s \leq m$ by

$$
\begin{aligned}
& P_{0}:=\mathbf{0}, \\
& P_{s}:=P_{s-1}+e_{s} t_{i_{s}}+\sum_{i_{s}<j}\left\{\left(a_{j}+\sum_{\substack{1 \leq l \leq s-1 \\
i_{l}=j}} e_{l}\right) e_{s} t_{j, i_{s}}\right\}
\end{aligned}
$$

for $1 \leq s \leq m$. Let $\overline{P_{s} P_{s+1}}$ be the path from $P_{s}$ to $P_{s+1}$ defined by a segment, and let $l_{w}\left(a_{1}, \ldots, a_{n}\right)$ be the polygonal path whose successive vertices are $P_{0}, P_{1}, \ldots, P_{m}$.

Lemma 3.1. As in the notation above, the vertex $P_{m}$ depends only on the integers $a_{1}, \ldots, a_{n}$ and the equivalence class of $w$ in $\Omega_{n}$.

Proof. For $w=a x_{i}^{e} x_{i}^{-e} b$, where $a, b \in \Omega_{n}$ and $e= \pm 1$, set $a=x_{i_{1}}^{e_{1}} x_{i_{2}}^{e_{2}} \cdots x_{i_{m^{\prime}}}^{e_{m^{\prime}}}$. If $e=1$, we have

$$
\begin{aligned}
& P_{m^{\prime}+1}=P_{m^{\prime}}+t_{i}+\sum_{i<j}\left\{\left(a_{j}+\sum_{\substack{1 \leq l \leq m^{\prime} \\
i_{l}=j}} e_{l}\right) t_{j, i}\right\}, \\
& P_{m^{\prime}+2}=P_{m^{\prime}+1}-t_{i}+\sum_{i<j}\left\{\left(a_{j}+\sum_{\substack{1 \leq l \leq m^{\prime} \\
i_{l}=j}} e_{l}\right) \cdot(-1) t_{j, i}\right\}=P_{m^{\prime}}, \\
& P_{s}=P_{s-2}, \quad s \geq m^{\prime}+3 .
\end{aligned}
$$

By an argument similar to the above, we obtain the required result for $e=-1$.

We denote $P_{m}$ above by $P_{w}\left(a_{1}, \ldots, a_{n}\right)$ for $w \in F_{n}$. In particular, $P_{1}\left(a_{1}, \ldots, a_{n}\right)$ $=\mathbf{0}$. It is clear that if $w=x_{1}^{w_{1}} x_{2}^{w_{2}} \cdots x_{n}^{w_{n}}$ in $H_{1}\left(F_{n}, \mathbf{Z}\right)$ then the $t_{i}$-coordinate of 
$P_{w}\left(a_{1}, \ldots, a_{n}\right)$ is $w_{i}$ for $1 \leq i \leq n$. If $w \in \Gamma_{n}(2), P_{w}\left(a_{1}, \ldots, a_{n}\right)$ also does not depend on $a_{1}, \ldots, a_{n}$. More precisely, we have

Lemma 3.2. As in the notation above, if $w \in \Gamma_{n}(2)$ and

$$
w=\left[x_{2}, x_{1}\right]^{w_{2,1}} \cdots\left[x_{n}, x_{n-1}\right]^{w_{n, n-1}} \in \mathcal{L}_{n}(2),
$$

then the $t_{i, j}$-coordinate of $P_{w}\left(a_{1}, \ldots, a_{n}\right)$ is $w_{i, j}$.

Proof. Set $w=x_{i_{1}}^{e_{1}} x_{i_{2}}^{e_{2}} \cdots x_{i_{m}}^{e_{m}}$, and take points $P_{0}, \ldots, P_{m}$ as above. For each $1 \leq s \leq m$, since the $t_{i, j}$-coordinate increase

$$
\delta_{j i_{s}}\left(a_{i}+\sum_{\substack{1 \leq r \leq s-1 \\ i_{r}=i}} e_{r}\right) e_{s}
$$

as a point moves from $P_{s-1}$ to $P_{s}$ where $\delta$ denotes the Kronecker delta, the $t_{i, j^{-}}$ coordinate of $P_{m}$ is

$$
\begin{aligned}
\sum_{1 \leq s \leq m} \delta_{j, i_{s}}\left(a_{i} e_{s}\right. & \left.+\sum_{\substack{1 \leq r \leq s-1 \\
i \\
i_{r}=i}} e_{r} e_{s}\right) \\
& =a_{i} \sum_{\substack{1 \leq s \leq m \\
j, i_{s}}} e_{s}+\sum_{1 \leq s \leq m} \delta_{j, i_{s}} \sum_{\substack{1 \leq r \leq s-1 \\
i_{r}=i}} e_{r} e_{s} .
\end{aligned}
$$

The first term is equal to zero since $w \in \Gamma_{n}(2)$. By considering rewriting $w$ as the $\bmod -\Gamma_{n}(3)$ normal form using the correcting process, we verify that the second term is nothing but $w_{i, j}$. This completes the proof of Lemma 3.2

Corollary 3.1. If $w \in \Gamma_{n}(3), P_{w}\left(a_{1}, \ldots, a_{n}\right)=\mathbf{0}$.

For any $P \in \mathbf{E}$, the translation function on $\mathbf{E}$ defined by

$$
t \mapsto t+P
$$

is denoted by $T_{P}$. By the definition of $l_{w}\left(a_{1}, \ldots, a_{n}\right)$, we see

Lemma 3.3. For $u, v \in \Omega_{n}, a_{1}, \ldots, a_{n} \in \mathbf{Z}$ and $u=x_{1}^{u_{1}} x_{2}^{u_{2}} \cdots x_{n}^{u_{n}}$ in $H_{1}\left(F_{n}, \mathbf{Z}\right)$,

$$
l_{u v}\left(a_{1}, \ldots, a_{n}\right)=l_{u}\left(a_{1}, \ldots, a_{n}\right) \cdot T_{P_{u}\left(a_{1}, \ldots, a_{n}\right)}\left(l_{v}\left(a_{1}+u_{1}, \ldots, a_{n}+u_{n}\right)\right) .
$$

Next, for any $w \in \Omega_{n}, a_{1}, \ldots, a_{n} \in \mathbf{Z}$ and a continuous real-valued function $f: \mathbf{E} \rightarrow \mathbf{R}$, we define integrations by

$$
I_{j}\left(f, w ; a_{1}, \ldots, a_{n}\right):=\int_{l_{w}\left(a_{1}, \ldots, a_{n}\right)} f(t) d t_{j} .
$$

Observing the proof of Lemma 3.1, we see that the integration $I_{j}\left(f, w ; a_{1}, \ldots, a_{n}\right)$ depends only on $f, a_{1}, \ldots, a_{n}$ and the equivalence class of $w$ in $\Omega_{n}$. Hence, from now on, we always consider $I_{j}\left(f, w ; a_{1}, \ldots, a_{n}\right)$ for $w \in F_{n}$. We remark that if $f: \mathbf{E} \rightarrow \mathbf{R}$ does not depend on the coordinates $t_{i, j}$ for any $1 \leq j<i \leq n$, then the integration $I_{j}\left(f, w ; a_{1}, \ldots, a_{n}\right)$ coincides with Chen's original integration $I_{j}(\bar{f}, w)$ for each $1 \leq j \leq n$, where $\bar{f}$ is the restriction of $f$ to the subspace $\mathbf{E}^{\prime}$ of $\mathbf{E}$ generated by the basis $x_{1}, \ldots, x_{n}$. In the following, if there is no confusion, we always write $f$ for $\bar{f}$ for simplicity. 
Here we recall a few properties of Chen's integration. For any continuous realvalued function $f, g: \mathbf{E}^{\prime} \rightarrow \mathbf{R}$, and $u, v, w \in F_{n}$, we have

$$
\begin{aligned}
& I_{j}(1, w)=w_{j} \quad \text { where } w=x_{1}^{w_{1}} \cdots x_{n}^{w_{n}} \in H_{1}\left(F_{n}, \mathbf{Z}\right), \\
& I_{j}(\alpha f+\beta g, w)=\alpha I_{j}(f, w)+\beta I_{j}(g, w), \quad \alpha, \beta \in \mathbf{R}, \\
& I_{j}(f, u v)=I_{j}(f, u)+I_{j}\left(f \circ T_{u}^{\prime}, v\right), \\
& I_{j}\left(f, u^{-1}\right)=-I_{j}\left(f \circ T_{u^{-1}}^{\prime}, u\right) .
\end{aligned}
$$

Here $T_{u}^{\prime}$ denotes the translation function on $\mathbf{E}^{\prime}$ defined by

$$
t^{\prime} \mapsto t^{\prime}+u_{1} t_{1}+\cdots+u_{n} t_{n}, \quad u=x_{1}^{u_{1}} \cdots x_{n}^{u_{n}} \in H_{1}\left(F_{n}, \mathbf{Z}\right) .
$$

(See [2] for basic materials concerning Chen's integration.)

Now, we consider some properties of the integration $I_{j}\left(f, w ; a_{1}, \ldots, a_{n}\right)$. By the linearity of the integration, we have

$$
I_{j}\left(\alpha f+\beta g, w ; a_{1}, \ldots, a_{n}\right)=\alpha I_{j}\left(f, w ; a_{1}, \ldots, a_{n}\right)+\beta I_{j}\left(g, w ; a_{1}, \ldots, a_{n}\right)
$$

for any $\alpha, \beta \in \mathbf{R}$.

Lemma 3.4. For $u, v \in F_{n}, a_{1}, \ldots, a_{n} \in \mathbf{Z}$, if $u=x_{1}^{u_{1}} x_{2}^{u_{2}} \cdots x_{n}^{u_{n}}$ in $H_{1}\left(F_{n}, \mathbf{Z}\right)$,

$$
\begin{aligned}
& I_{j}\left(f, u v ; a_{1}, \ldots, a_{n}\right) \\
& \quad=I_{j}\left(f, u ; a_{1}, \ldots, a_{n}\right)+I_{j}\left(f \circ T_{P_{u}\left(a_{1}, \ldots, a_{n}\right)}, v ; a_{1}+u_{1}, \ldots, a_{n}+u_{n}\right) .
\end{aligned}
$$

Proof. From Lemma 3.3, we see that

$$
\begin{aligned}
I_{j}\left(f, u v ; a_{1}, \ldots, a_{n}\right) & f(t) d t_{j} \\
& =\int_{l_{u v}\left(a_{1}, \ldots, a_{n}\right)} f(t) d t_{j} \\
& =\int_{l_{u}\left(a_{1}, \ldots, a_{n}\right) \cdot T_{P_{u}\left(a_{1}, \ldots, a_{n}\right)}\left(l_{v}\left(a_{1}+u_{1}, \ldots, a_{n}+u_{n}\right)\right)} f(t) d t_{j}+\int_{T_{P_{u}\left(a_{1}, \ldots, a_{n}\right)}\left(l_{v}\left(a_{1}+u_{1}, \ldots, a_{n}+u_{n}\right)\right)} f(t) d t_{j} .
\end{aligned}
$$

In the second term, if we consider the transformation of variables from $t$ to $t-$ $P_{u}\left(a_{1}, \ldots, a_{n}\right)$, we have

$$
\int_{T_{P_{u}\left(a_{1}, \ldots, a_{n}\right)}\left(l_{v}\left(a_{1}+u_{1}, \ldots, a_{n}+u_{n}\right)\right)} f(t) d t_{j}=\int_{l_{v}\left(a_{1}+u_{1}, \ldots, a_{n}+u_{n}\right)} f \circ T_{P_{u}\left(a_{1}, \ldots, a_{n}\right)}(t) d t_{j} .
$$

Hence,

$$
\begin{aligned}
& I_{j}\left(f, u v ; a_{1}, \ldots, a_{n}\right) \\
& \quad=I_{j}\left(f, u ; a_{1}, \ldots, a_{n}\right)+I_{j}\left(f \circ T_{P_{u}\left(a_{1}, \ldots, a_{n}\right)}, v ; a_{1}+u_{1}, \ldots, a_{n}+u_{n}\right) .
\end{aligned}
$$

This completes the proof of Lemma 3.4

As a corollary, we obtain

Corollary 3.2. For any $a_{1}, \ldots, a_{n} \in \mathbf{Z}, u \in F_{n}$ such that $u=x_{1}^{u_{1}} x_{2}^{u_{2}} \cdots x_{n}^{u_{n}} \in$ $H_{1}\left(F_{n}, \mathbf{Z}\right)$, and a real-valued function $f$ on $\mathbf{E}$, we have:

(1) $I_{j}\left(f, 1 ; a_{1}, \ldots, a_{n}\right)=0$.

(2) $I_{j}\left(f, u^{-1} ; a_{1}, \ldots, a_{n}\right)=-I_{j}\left(f \circ T_{P_{u^{-1}}\left(a_{1}, \ldots, a_{n}\right)}, u ; a_{1}-u_{1}, \ldots, a_{n}-u_{n}\right)$. 
(3) Furthermore, if $v \in F_{n}$ and $v=x_{1}^{v_{1}} x_{2}^{v_{2}} \cdots x_{n}^{v_{n}} \in H_{1}\left(F_{n}, \mathbf{Z}\right)$,

$$
\begin{aligned}
I_{j}(f,[u, v] & \left.; a_{1}, \ldots, a_{n}\right) \\
=I_{j} & \left(f, u ; a_{1}, \ldots, a_{n}\right)+I_{j}\left(f \circ T_{P_{u}\left(a_{1}, \ldots, a_{n}\right)}, v ; a_{1}+u_{1}, \ldots, a_{n}+u_{n}\right) \\
& -I_{j}\left(f \circ T_{P_{u v u^{-1}}\left(a_{1}, \ldots, a_{n}\right)}, u ; a_{1}+v_{1}, \ldots, a_{n}+v_{n}\right) \\
& -I_{j}\left(f \circ T_{P_{[u, v]}\left(a_{1}, \ldots, a_{n}\right)}, v ; a_{1}, \ldots, a_{n}\right) .
\end{aligned}
$$

Let $\mathbf{R}[t]$ be the commutative polynomial ring over $\mathbf{R}$ among indeterminates $t_{i}$ for $1 \leq i \leq n$ and $t_{i, j}$ for $1 \leq j<i \leq n$. Each element of $\mathbf{R}[t]$ is regarded as a realvalued function on $\mathbf{E}$ in a usual way. We consider the polynomial ring $\mathbf{R}\left[t_{1}, \ldots, t_{n}\right]$ as a subring of $\mathbf{R}[t]$. For any $f \in \mathbf{R}[t]$, we denote by $\operatorname{deg}(f)$ the degree of $f$.

Here we give some examples of calculations of the integrations. Clearly, for any $w \in F_{n}, I_{j}\left(1, w ; a_{1}, \ldots, a_{n}\right)=I_{j}(1, w)$ is the sum of the exponents of those $x_{j}$ which occur in $w$.

Lemma 3.5. (1) For any $p>q$,

$$
I_{j}\left(t_{i},\left[x_{p}, x_{q}\right] ; a_{1}, \ldots, a_{n}\right)= \begin{cases}\delta_{j q}, & i=p, \\ -\delta_{j p}, & i=q, \\ 0, & i \neq p, q .\end{cases}
$$

(2) For any $x \in \Gamma_{n}(3), I_{j}\left(t_{i}, x ; a_{1}, \ldots, a_{n}\right)=0$.

Proof. For part (1), let us consider the case where $i=p$. From (3) of Corollary 3.2, we have

$$
\begin{aligned}
I_{j}\left(t_{i},[\right. & \left.\left.x_{i}, x_{q}\right] ; a_{1}, \ldots, a_{n}\right) \\
= & I_{j}\left(t_{i}, x_{i} ; a_{1}, \ldots, a_{n}\right)+I_{j}\left(t_{i}+1, x_{q} ; a_{1}, \ldots, a_{i}+1, \ldots, a_{n}\right) \\
& \quad-I_{j}\left(t_{i}, x_{i} ; a_{1}, \ldots, a_{q}+1, \ldots, a_{n}\right)-I_{j}\left(t_{i}, x_{q} ; a_{1}, \ldots, a_{n}\right) \\
= & I_{j}\left(t_{i}, x_{i}\right)+I_{j}\left(t_{i}+1, x_{q}\right)-I_{j}\left(t_{i}, x_{i}\right)-I_{j}\left(t_{i}, x_{q}\right) \\
= & I_{j}\left(1, x_{q}\right)=\delta_{j q} .
\end{aligned}
$$

By an argument similar to the above, we obtain the other cases. The calculations are left to the reader for exercises.

For part (2), let us consider an element $[y, z] \in \Gamma_{n}(3)$ for $y \in \Gamma_{n}(2)$ and $z \in F_{n}$ such that $z=x_{1}^{z_{1}} \cdots x_{n}^{z_{n}} \in H_{1}\left(F_{n}, \mathbf{Z}\right)$. Then, from (3) of Corollary 3.2, we see that

$$
\begin{aligned}
I_{j}\left(t_{i},[\right. & {\left.[y, z] ; a_{1}, \ldots, a_{n}\right) } \\
& =I_{j}\left(t_{i}, y ; a_{1}, \ldots, a_{n}\right)-I_{j}\left(t_{i}+z_{i}, y ; a_{1}+z_{1}, \ldots, a_{n}+z_{n}\right) \\
& =I_{j}\left(t_{i}, y\right)-I_{j}\left(t_{i}+z_{i}, y\right)=-z_{i} I_{j}(1, y) \\
& =0 .
\end{aligned}
$$

Since $\Gamma_{n}(3)$ is generated by those elements $[y, z]$, we obtain the required result from Lemma 3.4 This completes the proof of Lemma 3.5

The following theorem is essentially due to Chen [2].

Theorem 3.1 (Chen [2]). Let $k \geq 2$ and $f \in \mathbf{R}\left[t_{1}, \ldots, t_{n}\right]$.

(1) If $w \in\left[\Gamma_{n}(2), \Gamma_{n}(2)\right]$, then $I_{j}\left(f, w ; a_{1}, \ldots, a_{n}\right)=0$. 
(2) If $w=\left[x_{i_{1}}, x_{i_{2}}, \ldots, x_{i_{k}}\right]$ and $\operatorname{deg}(f) \leq k-1$, then

$$
I_{j}\left(f, w ; a_{1}, \ldots, a_{n}\right)= \begin{cases}(-1)^{k-1} \alpha_{1}, & j=i_{1}, \\ (-1)^{k} \alpha_{2}, & j=i_{2}, \\ 0, & j \neq i_{1}, i_{2},\end{cases}
$$

where

$$
\alpha_{1}=\frac{\partial^{k-1} f}{\partial t_{i_{2}} \partial t_{i_{3}} \cdots \partial t_{i_{k}}}, \quad \alpha_{2}=\frac{\partial^{k-1} f}{\partial t_{i_{1}} \partial t_{i_{3}} \cdots \partial t_{i_{k}}} .
$$

Next, we consider a certain modification of (2) of the theorem above.

Lemma 3.6. Let $k \geq 5$ and $w=\left[x_{i_{1}}, \ldots, x_{i_{k-2}},\left[x_{i_{k-1}}, x_{i_{k}}\right]\right], i_{k-1}>i_{k}$, and let $f \in \mathbf{R}[t]$ such that

$$
f=g+g_{2,1} t_{2,1}+\cdots+g_{n, n-1} t_{n, n-1}
$$

for some $g, g_{i, j} \in \mathbf{R}\left[t_{1}, \ldots, t_{n}\right]$. Then

$$
I_{j}\left(f, w ; a_{1}, \ldots, a_{n}\right)=-I_{j}\left(\frac{\partial f}{\partial t_{i_{k-1}, i_{k}}}, w^{\prime} ; a_{1}, \ldots, a_{n}\right),
$$

where $w^{\prime}=\left[x_{i_{1}}, \ldots, x_{i_{k-2}}\right]$.

Proof. Using (3) of Corollary 3.2 we obtain

$$
\begin{aligned}
I_{j}\left(g, w ; a_{1}, \ldots, a_{n}\right) & \\
=I_{j} & \left(g, w^{\prime} ; a_{1}, \ldots, a_{n}\right)+I_{j}\left(g \circ T_{P_{w^{\prime}}\left(a_{1}, \ldots, a_{n}\right)},\left[x_{i_{k-1}}, x_{i_{k}}\right] ; a_{1}, \ldots, a_{n}\right) \\
& \quad-I_{j}\left(g \circ T_{P_{w^{\prime}\left[x_{i_{k-1},}, x_{i_{k}}\right] w^{\prime-1}}\left(a_{1}, \ldots, a_{n}\right)}, w^{\prime} ; a_{1}, \ldots, a_{n}\right) \\
& \quad-I_{j}\left(g \circ T_{P_{w}\left(a_{1}, \ldots, a_{n}\right)},\left[x_{i_{k-1}}, x_{i_{k}}\right] ; a_{1}, \ldots, a_{n}\right) .
\end{aligned}
$$

Since $w^{\prime}$ and $w \in \Gamma_{n}(3)$, we have

$$
P_{w^{\prime}}\left(a_{1}, \ldots, a_{n}\right)=P_{w}\left(a_{1}, \ldots, a_{n}\right)=\mathbf{0}
$$

and

$$
P_{w^{\prime}\left[x_{i_{k-1}}, x_{i_{k}}\right] w^{\prime-1}}\left(a_{1}, \ldots, a_{n}\right)=P_{\left[x_{i_{k-1}}, x_{i_{k}}\right]}\left(a_{1}, \ldots, a_{n}\right) .
$$

Since $g \in \mathbf{R}\left[t_{1}, \ldots, t_{n}\right]$, we see that

$$
g \circ T_{P_{w^{\prime}}\left(a_{1}, \ldots, a_{n}\right)}=g \circ T_{P_{w^{\prime}\left[x_{i_{k-1}}, x_{i_{k}}\right] w^{\prime}-1}\left(a_{1}, \ldots, a_{n}\right)}=g \circ T_{P_{w}\left(a_{1}, \ldots, a_{n}\right)}=g .
$$

Hence, $I_{j}\left(g, w ; a_{1}, \ldots, a_{n}\right)=0$.

By an argument similar to the above, for any $p>q$, we see that

$$
\begin{aligned}
I_{j}\left(g_{p, q} t_{p, q}, w ; a_{1}, \ldots, a_{n}\right) & \\
= & I_{j}\left(g_{p, q} t_{p, q}, w^{\prime} ; a_{1}, \ldots, a_{n}\right)+I_{j}\left(g_{p, q} t_{p, q},\left[x_{i_{k-1}}, x_{i_{k}}\right] ; a_{1}, \ldots, a_{n}\right) \\
& \quad-I_{j}\left(g_{p, q}\left(t_{p, q}+\delta_{(p, q),\left(i_{k-1}, i_{k}\right)}\right), w^{\prime} ; a_{1}, \ldots, a_{n}\right) \\
& \quad-I_{j}\left(g_{p, q} t_{p, q},\left[x_{i_{k-1}}, x_{i_{k}}\right] ; a_{1}, \ldots, a_{n}\right) \\
= & -\delta_{(p, q),\left(i_{k-1}, i_{k}\right)} I_{j}\left(g_{p, q}, w^{\prime} ; a_{1}, \ldots, a_{n}\right) .
\end{aligned}
$$

This completes the proof of Lemma 3.6, 
From Theorem 3.1 and Lemma 3.6, we obtain

Proposition 3.1. Let $k \geq 5$ and $w=\left[x_{i_{1}}, \ldots, x_{i_{k-2}},\left[x_{i_{k-1}}, x_{i_{k}}\right]\right], i_{k-1}>i_{k}$, and let $f \in \mathbf{R}[t]$ such that $\operatorname{deg}(f) \leq k-2$ and

$$
f=g+g_{2,1} t_{2,1}+\cdots+g_{n, n-1} t_{n, n-1}
$$

for some $g, g_{i, j} \in \mathbf{R}\left[t_{1}, \ldots, t_{n}\right]$. Then

$$
I_{j}\left(f, w ; a_{1}, \ldots, a_{n}\right)= \begin{cases}(-1)^{k-1} \beta_{1}, & j=i_{1}, \\ (-1)^{k} \beta_{2}, & j=i_{2}, \\ 0, & j \neq i_{1}, i_{2},\end{cases}
$$

where

$$
\beta_{1}=\frac{\partial^{k-2} f}{\partial t_{i_{k-1}, i_{k}} \partial t_{i_{2}} \partial t_{i_{3}} \cdots \partial t_{i_{k-2}}}, \quad \beta_{2}=\frac{\partial^{k-2} f}{\partial t_{i_{k-1}, i_{k}} \partial t_{i_{1}} \partial t_{i_{3}} \cdots \partial t_{i_{k-2}}} .
$$

Corollary 3.3. Using the same notation as that in Proposition 3.1, we have:

(1) If $\operatorname{deg}(f) \leq k-3$ and $f=g+g_{2,1} t_{2,1}+\cdots+g_{n, n-1} t_{n, n-1}$ for some $g, g_{i, j} \in$ $\mathbf{R}\left[t_{1}, \ldots, t_{n}\right]$, then $I_{j}\left(f, w ; a_{1}, \ldots, a_{n}\right)=0$.

(2) $I_{j}\left(t_{j_{1}} t_{j_{2}} \cdots t_{j_{k-3}} t_{p, q}, w ; a_{1}, \ldots, a_{n}\right) \neq 0$ if and only if

(i) $(p, q)=\left(i_{k-1} \cdot i_{k}\right)$,

(ii) $t_{j_{1}} \cdots t_{j_{k-3}} t_{j}=t_{i_{1}} \cdots t_{i_{k-2}}$,

(iii) $j=i_{1}$ or $j=i_{2}$.

\section{The structure of the Graded Quotients $\mathcal{L}_{n}^{N}(k)$}

In this section, we determine the group structure of the graded quotient $\mathcal{L}_{n}^{N}(k)$ of the lower central series of $F_{n}^{N}$. Set $K=\left[\Gamma_{n}(3), \Gamma_{n}(3)\right]\left[\left[\Gamma_{n}(2), \Gamma_{n}(2)\right], \Gamma_{n}(2)\right]$. If $k \leq 5$, we have $\mathcal{L}_{n}^{N}(k) \cong \mathcal{L}_{n}(k)$. Hence there is nothing to do anymore in this case. Consider a surjective homomorphism

$$
\iota_{k}: \mathcal{L}_{n}^{N}(k) \rightarrow \mathcal{L}_{n}^{M}(k)
$$

of abelian groups induced from a natural map $F_{n}^{N} \rightarrow F_{n}^{M}$. Since $\mathcal{L}_{n}^{M}(k)$ is a free abelian group due to Chen [2, if we denote by $\mathcal{K}_{n}(k)$ the kernel of $\iota_{k}$, we have

$$
\mathcal{L}_{n}^{N}(k) \cong \mathcal{K}_{n}(k) \oplus \mathcal{L}_{n}^{M}(k) .
$$

Hence it suffices to determine the group structure of $\mathcal{K}_{n}(k)$ for $k \geq 6$.

First, we have the natural isomorphisms

$$
\begin{aligned}
\mathcal{L}_{n}^{N}(k) & \cong \Gamma_{n}(k) K / \Gamma_{n}(k+1) K \\
\mathcal{L}_{n}^{M}(k) & \cong \Gamma_{n}(k)\left[\Gamma_{n}(2), \Gamma_{n}(2)\right] / \Gamma_{n}(k+1)\left[\Gamma_{n}(2), \Gamma_{n}(2)\right] .
\end{aligned}
$$

In general, for a group $F$ and its normal subgroups $G, H^{\prime}$ and $K^{\prime}$ such that $H^{\prime}$ is a subgroup of $G$, we have a natural isomorphism

$$
G K^{\prime} / H^{\prime} K^{\prime} \cong G / H^{\prime}\left(G \cap K^{\prime}\right) .
$$

Using (5), we see that

$$
\begin{aligned}
\mathcal{L}_{n}^{N}(k) & \cong \Gamma_{n}(k) / \Gamma_{n}(k+1)\left(\Gamma_{n}(k) \cap K\right), \\
\mathcal{L}_{n}^{M}(k) & \cong \Gamma_{n}(k) / \Gamma_{n}(k+1)\left(\Gamma_{n}(k) \cap\left[\Gamma_{n}(2), \Gamma_{n}(2)\right]\right) .
\end{aligned}
$$


Under these isomorphisms above, we verify that

$$
\begin{aligned}
\mathcal{K}_{n}(k) & \cong \Gamma_{n}(k+1)\left(\Gamma_{n}(k) \cap\left[\Gamma_{n}(2), \Gamma_{n}(2)\right]\right) / \Gamma_{n}(k+1)\left(\Gamma_{n}(k) \cap K\right) \\
& \cong \Gamma_{n}(k) \cap\left[\Gamma_{n}(2), \Gamma_{n}(2)\right] /\left(\Gamma_{n}(k) \cap K\right)\left(\Gamma_{n}(k+1) \cap\left[\Gamma_{n}(2), \Gamma_{n}(2)\right]\right) \\
& \cong\left(\Gamma_{n}(k) \cap\left[\Gamma_{n}(2), \Gamma_{n}(2)\right]\right) K /\left(\Gamma_{n}(k+1) \cap\left[\Gamma_{n}(2), \Gamma_{n}(2)\right]\right) K
\end{aligned}
$$

by using (5).

To determine the structure of $\mathcal{K}_{n}(k)$, we prepare a descending series of subgroups of $F_{n}$. For $k \geq 3$, denote by $\Theta_{n}(k)$ the subset of $F_{n}$ which consists of elements $w$ such that

$$
I_{j}\left(f, w ; a_{1}, \ldots, a_{n}\right)=0, \quad 1 \leq j \leq n
$$

for any $a_{1}, \ldots, a_{n} \in \mathbf{Z}$ and any $f \in \mathbf{R}[t]$ such that

$$
\operatorname{deg}(f) \leq k-3, \quad f=g+g_{2,1} t_{2,1}+\cdots+g_{n, n-1} t_{n, n-1}
$$

for some $g, g_{i, j} \in \mathbf{R}\left[t_{1}, \ldots, t_{n}\right]$. Then we have

$$
\Theta_{n}(3) \supset \Theta_{n}(4) \supset \Theta_{n}(5) \supset \cdots .
$$

Since $I_{j}\left(1, w ; a_{1}, \ldots, a_{n}\right)=I_{j}(1, w)$ is the sum of the exponents of those $x_{j}$ which occur in $w$, we see that $\Theta_{n}(3)=\Gamma_{n}(2)$. By Lemma 3.4 and (2) of Corollary 3.2, $\Theta_{n}(k)$ is a subgroup of $F_{n}$ for each $k \geq 3$. Furthermore, by (3) of Corollary 3.2. each of $\Theta_{n}(k)$ contains $\left[\Gamma_{n}(3), \Gamma_{n}(3)\right]$. Here we show that each of $\Theta_{n}(k)$ is a normal subgroup of $F_{n}$. First, we consider

Lemma 4.1. $\Theta_{n}(4) \subset \Gamma_{n}(3)$.

Proof. For any $w \in \Theta_{n}(4)$, since $w \in \Gamma_{n}(2)$, considering the mod- $\Gamma_{n}(3)$ normal form of $w$, we have

$$
w=\left[x_{2}, x_{1}\right]^{w_{2,1}} \cdots\left[x_{n}, x_{n-1}\right]^{w_{n, n-1}} \gamma
$$

for some $w_{i, j} \in \mathbf{Z}$ and $\gamma \in \Gamma_{n}(3)$. For any $1 \leq j<i \leq n$, from Lemmas 3.4 and 3.5, we see that

$$
\begin{aligned}
I_{j}\left(t_{i}, w ; a_{1}, \ldots, a_{n}\right)= & I_{j}\left(t_{i},\left[x_{2}, x_{1}\right]^{w_{2,1}} \ldots\left[x_{n}, x_{n-1}\right]^{w_{n, n-1}} ; a_{1}, \ldots, a_{n}\right) \\
& \quad+I_{j}\left(t_{i}, \gamma ; a_{1}, \ldots, a_{n}\right) \\
= & \sum_{r>s} w_{r, s} I_{j}\left(t_{i},\left[x_{r}, x_{s}\right] ; a_{1}, \ldots, a_{n}\right) \\
= & w_{i, j}=0 .
\end{aligned}
$$

This shows $w=\gamma \in \Gamma_{n}(3)$. This completes the proof of Lemma 4.1 .

For any $w \in \Theta_{n}(k), u \in F_{n}$ and $f \in \mathbf{R}[t]$ satisfying ([6), we have

$$
\begin{aligned}
& I_{j}\left(f, u w u^{-1} ; a_{1}, \ldots, a_{n}\right) \\
& =I_{j}\left(f, u ; a_{1}, \ldots, a_{n}\right)+I_{j}\left(f \circ T_{P_{u}\left(a_{1}, \ldots, a_{n}\right)}, w ; a_{1}+u_{1}, \ldots, a_{n}+u_{n}\right) \\
& -I_{j}\left(f \circ T_{P_{u w u^{-1}}\left(a_{1}, \ldots, a_{n}\right)}, u ; a_{1}, \ldots, a_{n}\right) \\
& =0
\end{aligned}
$$

since $u w u^{-1} \in \Gamma_{n}(3)$. Therefore $\Theta_{n}(k)$ is a normal subgroup of $F_{n}$.

Lemma 4.2. For $k \geq 3$, $\left[\left[\Gamma_{n}(2), \Gamma_{n}(2)\right], \Gamma_{n}(2)\right] \subset \Theta_{n}(k)$. 
Proof. Since $\left[\left[\Gamma_{n}(2), \Gamma_{n}(2)\right], \Gamma_{n}(2)\right]$ is normally generated by

$$
\left\{[x, y, z] \mid x, y, z \in \Gamma_{n}(2)\right\}
$$

in $F_{n}$, and since $\Theta_{n}(k)$ is a normal subgroup of $F_{n}$, it suffices to show that $[x, y, z] \in$ $\Theta_{n}(k)$ for $x, y, z \in \Gamma_{n}(2)$. For any $f \in \mathbf{R}[t]$ satisfying (6), using (3) of Corollary 3.2, we have

$$
\begin{aligned}
I_{j}\left(f,[x, y, z] ; a_{1}, \ldots, a_{n}\right) & \\
=I_{j}( & \left.f,[x, y] ; a_{1}, \ldots, a_{n}\right)+I_{j}\left(f \circ T_{\left.P_{[x, y]}\left(a_{1}, \ldots, a_{n}\right), z ; a_{1}, \ldots, a_{n}\right)}\right. \\
& \quad-I_{j}\left(f \circ T_{P_{[x, y] z[y, x]}\left(a_{1}, \ldots, a_{n}\right)},[x, y] ; a_{1}, \ldots, a_{n}\right) \\
& \quad-I_{j}\left(f \circ T_{P_{[x, y, z]}\left(a_{1}, \ldots, a_{n}\right)}, z ; a_{1}, \ldots, a_{n}\right) \\
= & I_{j}\left(f-f \circ T_{P_{[x, y] z[y, x]}\left(a_{1}, \ldots, a_{n}\right)},[x, y] ; a_{1}, \ldots, a_{n}\right) .
\end{aligned}
$$

On the other hand, if

$$
z=\left[x_{2}, x_{1}\right]^{z_{2,1}} \cdots\left[x_{n}, x_{n-1}\right]^{z_{n, n-1}} \in \mathcal{L}_{n}(3)
$$

for $z_{i, j} \in \mathbf{Z}$, we have

$$
P_{[x, y] z[y, x]}\left(a_{1}, \ldots, a_{n}\right)=z_{2,1} t_{2,1}+\cdots+z_{n, n-1} t_{n, n-1} .
$$

Hence if we set

$$
\begin{aligned}
g & :=f-f \circ T_{P_{[x, y] z[y, x]}\left(a_{1}, \ldots, a_{n}\right)} \\
& =z_{2,1} g_{2,1}+\cdots+z_{n, n-1} g_{n, n-1} \in \mathbf{R}\left[t_{1}, \ldots, t_{n}\right],
\end{aligned}
$$

then $I_{j}\left(g,[x, y] ; a_{1}, \ldots, a_{n}\right)=I_{j}(g,[x, y])=0$ since Chen's integration $I_{j}(g, \cdot)$ vanishes on $\left[\Gamma_{n}(2), \Gamma_{n}(2)\right]$ in general. This completes the proof of Lemma 4.2 .

Lemma 4.3. For $k \geq 5,\left[\Gamma_{n}(k-2), \Gamma_{n}(2)\right] \subset \Theta_{n}(k)$.

Proof. The group $\left[\Gamma_{n}(k-2), \Gamma_{n}(2)\right]$ is generated by $[u, v]$ for $u \in \Gamma_{n}(k-2)$ and $v \in \Gamma_{n}(2)$. For any $f \in \mathbf{R}[t]$ satisfying ([6), by an argument similar to that in Lemma 4.2 , we see that

$$
I_{j}\left(f,[u, v] ; a_{1}, \ldots, a_{n}\right)=I_{j}\left(g, u ; a_{1}, \ldots, a_{n}\right)=I_{j}(g, u)
$$

for some $g \in \mathbf{R}\left[t_{1}, \ldots, t_{n}\right]$ and $\operatorname{deg}(g) \leq k-4$. On the other hand, from Chen's result, we see that $I_{j}(g, u)=0$ since $u \in \Gamma_{n}(k-2)$. (See pages 150-151 in 2].) This completes the proof of Lemma 4.3 .

Lemma 4.4. For any $k \geq 5$ and $w \in\left[\Gamma_{n}(2), \Gamma_{n}(2)\right]$, there exists some $r \geq 1$ and $e_{1}, \ldots, e_{r} \in \mathbf{Z}$ such that

$$
w \equiv c_{1}^{e_{1}} \cdots c_{r}^{e_{r}} \quad\left(\bmod \left[\Gamma_{n}(k-2), \Gamma_{n}(2)\right]\right),
$$

where $c_{1}<\cdots<c_{r}$ are the basic commutators of $F_{n}$ which belong to $\left[\Gamma_{n}(2), \Gamma_{n}(2)\right]$.

Proof. In general, for any $y, z \in \Gamma_{n}(2)$, there exist some $y^{\prime}, z^{\prime} \in \Gamma_{n}(k-2)$, and $d_{i, j}, d_{i, j}^{\prime} \in \mathbf{Z}$ for $2 \leq i \leq k-1$ and $1 \leq j \leq m_{i}$ such that

$$
y=c_{2,1}^{d_{2,1}} \cdots c_{k-1, m_{k-1}}^{d_{k-1, m_{k-1}}} y^{\prime}, \quad z=c_{2,1}^{d_{2,1}^{\prime}} \cdots c_{k-1, m_{k-1}^{\prime}}^{d_{k-1}^{\prime}} z^{\prime} .
$$

Hence,

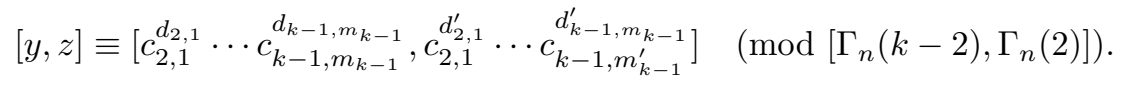


Since $\left[\Gamma_{n}(2), \Gamma_{n}(2)\right]$ is generated by $[y, z]$ for $y, z \in \Gamma_{n}(2)$, we see that any $w \in$ $\left[\Gamma_{n}(2), \Gamma_{n}(2)\right]$ can be written as

$$
w \equiv \bar{c}_{1}^{e_{1}^{\prime}} \cdots \bar{c}_{s}^{e_{s}^{\prime}} \quad\left(\bmod \left[\Gamma_{n}(k-2), \Gamma_{n}(2)\right]\right)
$$

where the $\bar{c}_{i}$ are the basic commutators in $\Gamma_{n}(2)$.

Then if we apply Hall's correcting process to $w^{\prime}:=\bar{c}_{1}^{e_{1}^{\prime}} \ldots \bar{c}_{s}^{e_{s}^{\prime}}$ to obtain the $\bmod -\Gamma_{n}(2 k-4)$ normal form, we have

$$
w^{\prime}=c_{1}^{e_{1}} \cdots c_{r}^{e_{r}} \gamma
$$

where all the $c_{i}$ belong to $\left[\Gamma_{n}(2), \Gamma_{n}(2)\right]$, and $\gamma$ is a product of the commutators $\left[u_{1}, u_{2}, \ldots, u_{t}\right] \in \Gamma_{n}(2 k-4)$ and each element $u_{i}$ of the component is in $\Gamma_{n}(2)$. Since such commutators belong to $\left[\Gamma_{n}(k-2), \Gamma_{n}(2)\right]$, so does $\gamma$. This completes the proof of Lemma 4.4 .

Lemma 4.5. For $k \geq 5, \Gamma_{n}(k) \cap\left[\Gamma_{n}(2), \Gamma_{n}(2)\right] \subset\left[\Gamma_{n}(k-2), \Gamma_{n}(2)\right]\left[\Gamma_{n}(3), \Gamma_{n}(3)\right]$.

Proof. For any $w \in \Gamma_{n}(k) \cap\left[\Gamma_{n}(2), \Gamma_{n}(2)\right]$, we see

$$
w \equiv c_{1}^{e_{1}} \cdots c_{r}^{e_{r}} \quad\left(\bmod \left[\Gamma_{n}(k-2), \Gamma_{n}(2)\right]\right)
$$

for basic commutators $c_{1}<\cdots<c_{r}$ of $F_{n}$ which belong to $\left[\Gamma_{n}(2), \Gamma_{n}(2)\right]$ from Lemma 4.4. Since $w \in \Gamma_{n}(k)$, we may assume the weight of $c_{i}$ is greater than $k-1$ for each $1 \leq i \leq r$. On the other hand, such basic commutators belong to $\left[\Gamma_{n}(k-2), \Gamma_{n}(2)\right]$ or $\left[\Gamma_{n}(3), \Gamma_{n}(3)\right]$. This completes the proof of Lemma 4.5.

From Lemmas 4.3 and 4.5. we see that for each $k \geq 5$,

$$
\Gamma_{n}(k) \cap\left[\Gamma_{n}(2), \Gamma_{n}(2)\right] \subset \Theta_{n}(k) .
$$

Using this, we can determine the group structure of $\mathcal{K}_{n}(k)$. Set

$$
\mathfrak{E}:=\left\{\left[x_{i_{1}}, \ldots, x_{i_{k-2}},\left[x_{i_{k-1}}, x_{i_{k}}\right]\right] \mid i_{1}>i_{2} \leq i_{3} \leq \cdots \leq i_{k-2}, i_{k-1}>i_{k}\right\} .
$$

Theorem 4.1. For $k \geq 6, \mathcal{K}_{n}(k)$ is a free abelian group with basis $\mathfrak{E}$.

Proof. For any $x \in \Gamma_{n}(k) \cap\left[\Gamma_{n}(2), \Gamma_{n}(2)\right]$, we have

$$
x=c_{1}^{e_{1}} \cdots c_{r}^{e_{r}} x^{\prime}
$$

for some basic commutators $c_{1}<\cdots<c_{r}$ of weight $k$, and $x^{\prime} \in \Gamma_{n}(k+1)$. Since $x \in\left[\Gamma_{n}(2), \Gamma_{n}(2)\right]$, observing the image of $x$ by the natural map $\mathcal{L}_{n}(k) \rightarrow \mathcal{L}_{n}^{M}(k)$, we may assume that $c_{i} \in\left[\Gamma_{n}(2), \Gamma_{n}(2)\right]$ for $1 \leq i \leq r$. Hence $x^{\prime} \in\left[\Gamma_{n}(2), \Gamma_{n}(2)\right]$, and each of the $c_{i}$ belongs to $\left[\Gamma_{n}(3), \Gamma_{n}(3)\right],\left[\left[\Gamma_{n}(2), \Gamma_{n}(2)\right], \Gamma_{n}(2)\right]$ or $\mathfrak{E}$ since $k \geq 6$. This shows that $\mathfrak{E}$ generates $\mathcal{K}_{n}(k)$. Set

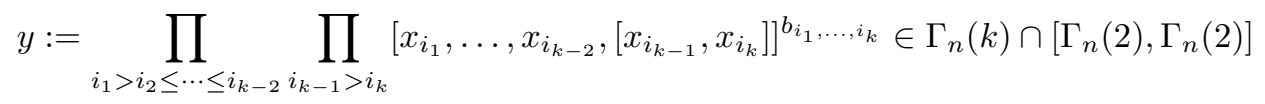

for $b_{i_{1}, \ldots, i_{k}} \in \mathbf{Z}$, and suppose $y=1 \in \mathcal{K}_{n}(k)$.

Now, for any $j_{1}>j_{2} \leq j_{3} \leq \cdots \leq j_{k-2}$ and $j_{k-1}>j_{k}$, consider

$$
g:=t_{j_{2}} \cdots t_{j_{k-2}} t_{j_{k-1}, j_{k}} \in \mathbf{R}[t] .
$$

Since $\operatorname{deg}(g)=k-2$ and $x \in \Theta_{n}(k+1)$, for any $a_{1}, \ldots, a_{n}$, we have

$$
0=I_{j_{1}}\left(g, x ; a_{1}, \ldots, a_{n}\right)=(-1)^{k-1} b_{j_{1}, \ldots, j_{k}} \frac{\partial^{k-3}\left(t_{j_{2}} \cdots t_{j_{k-2}}\right)}{\partial t_{j_{2}} \cdots \partial t_{j_{k-2}}}
$$


from Proposition 3.1. Since

$$
\frac{\partial^{k-3}\left(t_{j_{2}} \cdots t_{j_{k-2}}\right)}{\partial t_{j_{2}} \cdots \partial t_{j_{k-2}}} \neq 0
$$

we obtain $b_{j_{1}, \ldots, j_{k}}=0$. This shows that $\mathfrak{E}$ is linearly independent. This completes the proof of Theorem 4.1

Corollary 4.1. For $k \geq 6$,

$$
\operatorname{rank}_{\mathbf{Z}}\left(\mathcal{K}_{n}(k)\right)=\frac{1}{2} n(n-1)(k-3)\left(\begin{array}{c}
n+k-4 \\
k-2
\end{array}\right)
$$

and

$$
\operatorname{rank}_{\mathbf{Z}}\left(\mathcal{L}_{n}^{N}(k)\right)=(k-1)\left(\begin{array}{c}
k+n-2 \\
k
\end{array}\right)+\frac{1}{2} n(n-1)(k-3)\left(\begin{array}{c}
n+k-4 \\
k-2
\end{array}\right) .
$$

\section{An Application to the STUdy of the Johnson homomorphisms}

In this section, we consider a reduction of the target of the Johnson homomorphism $\tau_{k}^{\prime}$ to $H^{*} \otimes \mathbf{z} \mathcal{L}_{n}^{N}(k+1)$. Let

$$
\tau_{k, N}^{\prime}: \operatorname{gr}^{k}\left(\mathcal{A}_{n}^{\prime}\right) \rightarrow H^{*} \otimes_{\mathbf{Z}} \mathcal{L}_{n}^{N}(k+1)
$$

be the composition of $\tau_{k}^{\prime}$ and the natural projection $H^{*} \otimes_{\mathbf{z}} \mathcal{L}_{n}(k+1) \rightarrow H^{*} \otimes_{\mathbf{z}}$ $\mathcal{L}_{n}^{N}(k+1)$. It is easily seen that $\tau_{k, N}^{\prime}$ is a $\operatorname{GL}(n, \mathbf{Z})$-equivariant homomorphism.

In the following we study the cokernel of $\left(\tau_{k, N}^{\prime}\right)_{\mathbf{Q}}$ for $n \geq k+2$. In particular, we show that there is an obstruction $H_{\mathbf{Q}}^{\left[k-2,1^{2}\right]}$ for the surjectivity of $\tau_{k, \mathbf{Q}}^{\prime}$, and that it also appears in $\operatorname{Coker}\left(\left(\tau_{k, N}^{\prime}\right)_{\mathbf{Q}}\right)$. Finally, we conclude that the $\mathrm{GL}(n, \mathbf{Z})$-irreducible decomposition of $\operatorname{Coker}\left(\left(\tau_{k, N}^{\prime}\right)_{\mathbf{Q}}\right)$ is $S^{k} H_{\mathbf{Q}} \oplus H_{\mathbf{Q}}^{\left[k-2,1^{2}\right]}$ for $n \geq k+2$.

5.1. The image of $\tau_{k}^{\prime}$. In the next subsection, we detect $H_{\mathbf{Q}}^{\left[k-2,1^{2}\right]}$ in $\operatorname{Coker}\left(\tau_{k, \mathbf{Q}}^{\prime}\right)$ using trace maps. To do this, we prepare a finitely generated submodule of $H^{*} \otimes \mathbf{z}$ $\mathcal{L}_{n}(k+1)$ which contains $\operatorname{Im}\left(\tau_{k}^{\prime}\right)$. Let $V_{n}(k)$ be a submodule of $H^{*} \otimes_{\mathbf{z}} \mathcal{L}_{n}(k+1)$ generated by

(A1): $x_{i}^{*} \otimes[A, B]$,

(A2): $x_{i}^{*} \otimes[A, B, C]$,

(A3): $x_{i}^{*} \otimes\left[x_{i_{1}}, x_{i_{2}}, x_{i_{3}}, \ldots, x_{i_{k+1}}\right]$,

(A4): $x_{i}^{*} \otimes\left[x_{i}, x_{i_{2}}, \ldots, x_{i_{k+1}}\right]-x_{j}^{*} \otimes\left[x_{j}, x_{i_{3}}, \ldots, x_{i_{k+1}}, x_{i_{2}}\right]$,

(A5): $x_{i}^{*} \otimes\left[x_{i}, x_{i_{2}}, \ldots, x_{i_{k+1}}\right]-x_{i}^{*} \otimes\left[x_{i}, x_{i_{3}}, \ldots, x_{i_{k+1}}, x_{i_{2}}\right]$,

(A6): $x_{i}^{*} \otimes\left[x_{i_{1}}, x_{i_{2}}, x_{i}, x_{i_{4}}, \ldots, x_{i_{k+1}}\right]-x_{i}^{*} \otimes\left[x_{i_{1}}, x_{i_{2}}, x_{i}, x_{i_{5}} \ldots, x_{i_{k+1}}, x_{i_{4}}\right]$,

(A7): $x_{i}^{*} \otimes\left[x_{i_{1}}, x_{i_{2}}, x_{i}, x_{i_{4}}, x_{i_{5}}, x_{i_{6}}, \ldots, x_{i_{k+1}}\right]$

$-x_{i}^{*} \otimes\left[x_{i_{1}}, x_{i_{2}}, x_{i}, x_{i_{5}}, x_{i_{4}}, x_{i_{6}}, \ldots, x_{i_{k+1}}\right]$,

(A8): $x_{i}^{*} \otimes\left[x_{i}, x_{i_{2}}, x_{i}, x_{i_{4}}, \ldots, x_{i_{k+1}}\right]-x_{j}^{*} \otimes\left[x_{j}, x_{i_{2}}, x_{i}, x_{i_{4}}, \ldots, x_{i_{k+1}}\right]$

$$
-x_{j}^{*} \otimes\left[x_{i}, x_{i_{2}}, x_{j}, x_{i_{4}}, \ldots, x_{i_{k+1}}\right],
$$

where $A, B, C$ and the indices $1 \leq i, j, i_{l} \leq n$ satisfy the conditions

(A1): $\operatorname{wt}(A), \operatorname{wt}(B) \geq 3$ and $\operatorname{wt}(A)+\operatorname{wt}(B)=k+1$,

(A2): $\operatorname{wt}(A), \operatorname{wt}(B), \operatorname{wt}(C) \geq 2$ and $\operatorname{wt}(A)+\operatorname{wt}(B)+\operatorname{wt}(C)=k+1$,

(A3): $i \neq i_{1}, i_{2}, i_{3}$,

(A4): $i \neq i_{2}, i_{3}, j$ and $j \neq i_{3}, i_{4}$,

(A5): $i \neq i_{2}, i_{3}, i_{4}$, 
(A6), (A7): $i \neq i_{1}, i_{2}$,

(A8): $i \neq j, i_{2}$ and $j \neq i_{2}$,

respectively. We do not consider (A1) and (A2) for $k<5$. In this subsection, we use $\equiv$ for the equality in the quotient module of $H^{*} \otimes \mathbf{z} \mathcal{L}_{n}(k+1)$ by $V_{n}(k)$. Then we show

Theorem 5.1. For $k \geq 1$ and $n \geq 6, \operatorname{Im}\left(\tau_{k}^{\prime}\right) \subset V_{n}(k)$.

Before showing Theorem [5.1, we prepare

Lemma 5.1. For any $n \geq 3$, we have:

(1) For any $i \neq i_{1}, i_{2}$,

$$
\begin{aligned}
& x_{i}^{*} \otimes\left[x_{i_{1}}, x_{i_{2}}, x_{i}, x_{i_{4}}, \ldots, x_{i_{k+1}}\right] \\
& \quad=x_{i}^{*} \otimes\left[x_{i}, x_{i_{2}}, x_{i_{1}}, x_{i_{4}}, \ldots, x_{i_{k+1}}\right]-x_{i}^{*} \otimes\left[x_{i}, x_{i_{1}}, x_{i_{2}}, x_{i_{4}}, \ldots, x_{i_{k+1}}\right] .
\end{aligned}
$$

(2) For any $i, j \neq i_{1}, i_{2}$ and $\sigma \in \mathfrak{S}_{k-2}$,

$$
x_{i}^{*} \otimes\left[x_{i_{1}}, x_{i_{2}}, x_{i}, x_{j_{1}}, \ldots, x_{j_{k-2}}\right] \equiv x_{j}^{*} \otimes\left[x_{i_{1}}, x_{i_{2}}, x_{j}, x_{\sigma\left(j_{1}\right)} \ldots, x_{\sigma\left(j_{k-2}\right)}\right] .
$$

(3) If $n \geq 6$, for any $i, j \neq i_{2}, i_{3}, i_{4}$ and $i \neq j$,

$$
x_{i}^{*} \otimes\left[x_{i}, x_{i_{2}}, \ldots, x_{i_{k+1}}\right] \equiv x_{j}^{*} \otimes\left[x_{j}, x_{i_{2}}, \ldots, x_{i_{k+1}}\right] .
$$

Proof of Lemma 5.1. Part (1) is immediately obtained from the Jacobi identity

$$
\left[x_{i_{1}}, x_{i_{2}}, x_{i}\right]=\left[x_{i}, x_{i_{2}}, x_{i_{1}}\right]-\left[x_{i}, x_{i_{1}}, x_{i_{2}}\right] .
$$

For part (2), if $j=i$, it is obtained from (A6) and (A7). If not, we have

$$
\begin{aligned}
x_{i}^{*} \otimes & {\left[x_{i_{1}}, x_{i_{2}}, x_{i}, x_{j_{1}}, \ldots, x_{j_{k-2}}\right] } \\
& \stackrel{(1)}{=} x_{i}^{*} \otimes\left[x_{i}, x_{i_{2}}, x_{i_{1}}, x_{i_{4}}, \ldots, x_{i_{k+1}}\right]-x_{i}^{*} \otimes\left[x_{i}, x_{i_{1}}, x_{i_{2}}, x_{i_{4}}, \ldots, x_{i_{k+1}}\right] \\
& \stackrel{(\mathbf{A} 4)}{=} x_{j}^{*} \otimes\left[x_{j}, x_{i_{1}}, x_{i_{4}}, \ldots, x_{i_{k+1}}, x_{i_{2}}\right]-x_{j}^{*} \otimes\left[x_{j}, x_{i_{2}}, x_{i_{4}}, \ldots, x_{i_{k+1}}, x_{i_{1}}\right] \\
& \stackrel{(\mathbf{A} \mathbf{A})}{=} x_{j}^{*} \otimes\left[x_{j}, x_{i_{2}}, x_{i_{1}}, x_{i_{4}}, \ldots, x_{i_{k+1}}\right]-x_{j}^{*} \otimes\left[x_{j}, x_{i_{1}}, x_{i_{2}}, x_{i_{4}}, \ldots, x_{i_{k+1}}\right] \\
& \stackrel{(1)}{=} x_{j}^{*} \otimes\left[x_{i_{1}}, x_{i_{2}}, x_{j}, x_{j_{1}}, \ldots, x_{j_{k-2}}\right] .
\end{aligned}
$$

Hence we obtain part (2).

For part (3), we can take some $1 \leq k \leq n$ such that $k \neq i, j, i_{2}, i_{3}, i_{4}$. Then we see that

$$
x_{i}^{*} \otimes\left[x_{i}, x_{i_{2}}, \ldots, x_{i_{k+1}}\right] \equiv x_{k}^{*} \otimes\left[x_{k}, x_{i_{3}}, \ldots, x_{i_{k+1}}, x_{i_{2}}\right] \equiv x_{j}^{*} \otimes\left[x_{j}, x_{i_{2}}, \ldots, x_{i_{k+1}}\right]
$$

by (A4). This completes of the proof of Lemma 5.1

Proof of Theorem [5.1. We prove this theorem by induction on $k$. For $k=1$, since $\operatorname{gr}^{1}\left(\mathcal{A}_{n}\right)=\mathrm{IA}_{n}^{\mathrm{ab}}$ is generated by $K_{i j}$ and $K_{i j l}$, it is clear from (44). Assume $k \geq 1$. Since

$$
\tau^{\prime}=\bigoplus_{k \geq 1} \tau_{k}^{\prime}: \operatorname{gr}\left(\mathcal{A}_{n}^{\prime}\right) \rightarrow \operatorname{Der}\left(\mathcal{L}_{n}\right)
$$

is a Lie algebra homomorphism, it suffices to show that $\left[(\mathbf{A} \mathbf{1}), \tau_{1}\left(K_{p q}\right)\right], \ldots,[(\mathbf{A} \mathbf{8})$, $\left.\tau_{1}\left(K_{p q}\right)\right]$ and $\left[(\mathbf{A} \mathbf{1}), \tau_{1}\left(K_{p q r}\right)\right], \ldots,\left[(\mathbf{A} \mathbf{8}), \tau_{1}\left(K_{p q r}\right)\right]$ belong to $V_{n}(k+1)$ for any $p, q$ and $r$. We show this by direct computation. Here we give some examples of it. 
Step I. [(A1), $\left.\tau_{1}\left(K_{p q}\right)\right]$.

Observe

$$
\begin{aligned}
{\left[x_{i}^{*} \otimes[A, B], \tau_{1}\left(K_{p q}\right)\right] } & \\
= & x_{i}^{*} \otimes\left[A^{\partial \tau_{1}\left(K_{p q}\right)}, B\right]+x_{i}^{*} \otimes\left[A, B^{\partial \tau_{1}\left(K_{p q}\right)}\right]-\delta_{i, p} x_{p}^{*} \otimes\left[[A, B], x_{q}\right] \\
& \quad-\delta_{i, q} x_{p}^{*} \otimes\left[x_{p},[A, B]\right] .
\end{aligned}
$$

By the Jacobi identity, we have

$\left[[A, B], x_{q}\right]=-\left[\left[B, x_{q}\right], A\right]-\left[\left[x_{q}, A\right], B\right], \quad\left[x_{p},[A, B]\right]=-\left[A,\left[B, x_{p}\right]\right]-\left[B,\left[x_{p}, A\right]\right]$.

Hence $\left[(\mathbf{A} \mathbf{1}), \tau_{1}\left(K_{p q}\right)\right] \in V_{n}(k+1)$. Similarly, we see $\left[(\mathbf{A} \mathbf{1}), \tau_{1}\left(K_{p q r}\right)\right] \in V_{n}(k+1)$.

Step II. $\left[(\mathbf{A 2}), \tau_{1}\left(K_{p q}\right)\right]$.

Observe

$$
\begin{aligned}
{\left[x_{i}^{*} \otimes[A, B, C], \tau_{1}\left(K_{p q}\right)\right] } & \\
= & x_{i}^{*} \otimes\left[A^{\partial \tau_{1}\left(K_{p q}\right)}, B, C\right]+x_{i}^{*} \otimes\left[A, B^{\partial \tau_{1}\left(K_{p q}\right)}, C\right]+x_{i}^{*} \otimes\left[A, B, C^{\partial \tau_{1}\left(K_{p q}\right)}\right] \\
& -\delta_{i, p} x_{p}^{*} \otimes\left[[A, B, C], x_{q}\right]-\delta_{i, q} x_{p}^{*} \otimes\left[x_{p},[A, B, C]\right] .
\end{aligned}
$$

By the Jacobi identity, we have

$$
\begin{aligned}
{\left[[A, B, C], x_{q}\right] } & =-\left[\left[C, x_{q}\right],[A, B]\right]-\left[\left[x_{q},[A, B]\right], C\right] \\
& =\left[A, B,\left[C, x_{q}\right]\right]+\left[A,\left[B, x_{q}\right], C\right]+\left[B,\left[x_{q}, A\right], C\right], \\
{\left[x_{p},[A, B, C]\right] } & =-\left[A, B,\left[C, x_{p}\right]\right]-\left[A,\left[B, x_{p}\right], C\right]-\left[B,\left[x_{p}, A\right], C\right] .
\end{aligned}
$$

Hence $\left[(\mathbf{A 2}), \tau_{1}\left(K_{p q}\right)\right] \in V_{n}(k+1)$. Similarly, we see $\left[(\mathbf{A 2}), \tau_{1}\left(K_{p q r}\right)\right] \in V_{n}(k+1)$.

Step III. [(A3), $\left.\tau_{1}\left(K_{p q}\right)\right]$.

In

$$
\begin{aligned}
& {\left[(\mathbf{A 3}), \tau_{1}\left(K_{p q}\right)\right]} \\
& =\delta_{i_{1}, p} x_{i}^{*} \otimes\left[x_{i_{1}}, x_{q}, x_{i_{2}}, x_{i_{3}}, \ldots, x_{i_{k+1}}\right]+\delta_{i_{2}, p} x_{i}^{*} \otimes\left[x_{i_{1}},\left[x_{i_{2}}, x_{q}\right], x_{i_{3}}, \ldots, x_{i_{k+1}}\right] \\
& \quad+\delta_{i_{3}, p} x_{i}^{*} \otimes\left[x_{i_{1}}, x_{i_{2}},\left[x_{i_{3}}, x_{q}\right], x_{i_{4}}, \ldots, x_{i_{k+1}}\right] \\
& \quad+\sum_{l=4}^{k+1} \delta_{i_{l}, p} \underline{x_{i}^{*} \otimes\left[x_{i_{1}}, x_{i_{2}}, x_{i_{3}}, x_{i_{4}}, \ldots, x_{i_{l-1}},\left[x_{i_{l}}, x_{q}\right], x_{i_{l+1}}, \ldots, x_{i_{k+1}}\right]_{1}} \\
& \quad-\delta_{i, p} \underline{x_{i}^{*} \otimes\left[x_{i_{1}}, x_{i_{2}}, x_{i_{3}}, \ldots, x_{i_{k+1}}, x_{q}\right]_{\text {(2) }}-\delta_{i, q} x_{p}^{*} \otimes\left[x_{p},\left[x_{i_{1}}, x_{i_{2}}, x_{i_{3}}, \ldots, x_{i_{k+1}}\right]\right],}
\end{aligned}
$$

(2) $\equiv 0$ by (A3). On the other hand, using the Jacobi identity

$$
\left[X,\left[x_{a}, x_{b}\right]\right]=\left[X, x_{a}, x_{b}\right]-\left[X, x_{b}, x_{a}\right],
$$

we see (1) $\equiv 0$ by $(\mathbf{A} 3)$. If $q \neq i$, we see $\left[(\mathbf{A} \mathbf{3}), \tau_{1}\left(K_{p q}\right)\right] \equiv 0$ since all terms other than (1) and (2) in the equation above are of type (A3). Hence, consider the case where $q=i$.

Suppose $p=i_{1}$. If $i_{3} \neq i_{1}$, we have

$$
\begin{aligned}
& {\left[(\mathbf{A 3}), \tau_{1}\left(K_{p q}\right)\right]} \\
& \quad \equiv-x_{i}^{*} \otimes\left[x_{i}, x_{i_{1}}, x_{i_{2}}, x_{i_{3}}, \ldots, x_{i_{k+1}}\right]+x_{i_{1}}^{*} \otimes\left[x_{i_{1}}, x_{i_{2}}, x_{i_{3}}, \ldots, x_{i_{k+1}}, x_{i_{1}}\right] \equiv 0
\end{aligned}
$$


by (A4). If $i_{3}=i_{1}$, using (A5), (A6) and (A8), we have

$$
\begin{aligned}
& {\left[(\mathbf{A} \mathbf{3}), \tau_{1}\left(K_{p q}\right)\right]} \\
& \equiv-x_{i}^{*} \otimes\left[x_{i}, x_{i_{1}}, x_{i_{2}}, x_{i_{3}}, x_{i_{4}}, \ldots, x_{i_{k+1}}\right]-x_{i}^{*} \otimes\left[x_{i_{1}}, x_{i_{2}}, x_{i}, x_{i_{3}}, x_{i_{4}}, \ldots, x_{i_{k+1}}\right] \\
& \quad+x_{i_{1}}^{*} \otimes\left[x_{i_{1}}, x_{i_{2}}, x_{i_{1}}, x_{i_{4}}, \ldots, x_{i_{k+1}}, x_{i_{1}}\right] \\
& \equiv-x_{i}^{*} \otimes\left[x_{i}, x_{i_{2}}, x_{i_{3}}, x_{i_{4}}, \ldots, x_{i_{k+1}}, x_{i_{1}}\right]-x_{i}^{*} \otimes\left[x_{i_{1}}, x_{i_{2}}, x_{i}, x_{i_{4}}, \ldots, x_{i_{k+1}}, x_{i_{3}}\right] \\
& \quad+x_{i_{1}}^{*} \otimes\left[x_{i_{1}}, x_{i_{2}}, x_{i_{1}}, x_{i_{4}}, \ldots, x_{i_{k+1}}, x_{i_{1}}\right] \\
& \equiv 0 .
\end{aligned}
$$

Similarly, we see $\left[(\mathbf{A} 3), \tau_{1}\left(K_{p q}\right)\right] \equiv 0$ for $p=i_{2}$. Suppose $p=i_{3}$ and $p \neq i_{1}, i_{2}$. By (A6), we have

$$
\begin{aligned}
& {\left[(\mathbf{A} \mathbf{3}), \tau_{1}\left(K_{p q}\right)\right]} \\
& \quad \equiv-x_{i}^{*} \otimes\left[x_{i_{1}}, x_{i_{2}}, x_{i}, x_{i_{3}}, x_{i_{4}}, \ldots, x_{i_{k+1}}\right]+x_{i_{3}}^{*} \otimes\left[x_{i_{1}}, x_{i_{2}}, x_{i_{3}}, x_{i_{4}}, \ldots, x_{i_{k+1}}, x_{i_{3}}\right] \\
& \quad \equiv 0
\end{aligned}
$$

Therefore we have $\left[(\mathbf{A} \mathbf{3}), \tau_{1}\left(K_{p q}\right)\right] \in V_{n}(k+1)$ for any cases. Similarly, we obtain $\left[(\mathbf{A 3}), \tau_{1}\left(K_{p q r}\right)\right] \in V_{n}(k+1)$.

Step IV. $\left[(\mathbf{A 6}), \tau_{1}\left(K_{p q}\right)\right]$.

In

$$
\begin{aligned}
& {\left[(\mathbf{A 6}), \tau_{1}\left(K_{p q}\right)\right]} \\
& \equiv \delta_{i_{1}, p}\left(x_{i}^{*} \otimes\left[x_{i_{1}}, x_{q}, x_{i_{2}}, x_{i}, x_{i_{4}}, \ldots, x_{i_{k+1}}\right]-x_{i}^{*} \otimes\left[x_{i_{1}}, x_{q}, x_{i_{2}}, x_{i}, x_{i_{5}}, \ldots, x_{i_{k+1}}, x_{i_{4}}\right]\right) \\
& +\delta_{i_{2}, p}\left(x_{i}^{*} \otimes\left[x_{i_{1}},\left[x_{i_{2}}, x_{q}\right], x_{i}, x_{i_{4}}, \ldots, x_{i_{k+1}}\right]-x_{i}^{*} \otimes\left[x_{i_{1}},\left[x_{i_{2}}, x_{q}\right], x_{i}, x_{i_{5}}, \ldots, x_{i_{k+1}}, x_{i_{4}}\right]\right) \\
& +\delta_{i, p}\left(\frac{x_{i}^{*} \otimes\left[x_{i_{1}}, x_{i_{2}},\left[x_{i}, x_{q}\right], x_{i_{4}}, \ldots, x_{i_{k+1}}\right]-x_{i}^{*} \otimes\left[x_{i_{1}}, x_{i_{2}},\left[x_{i}, x_{q}\right], x_{i_{5}}, \ldots, x_{i_{k+1}}, x_{i_{4}}\right]_{1}}{1}\right) \\
& +\delta_{i_{4}, p}\left(\underline{\left(x_{i}^{*} \otimes\left[x_{i_{1}}, x_{i_{2}}, x_{i},\left[x_{i_{4}}, x_{q}\right], \ldots, x_{i_{k+1}}\right]-x_{i}^{*} \otimes\left[x_{i_{1}}, x_{i_{2}}, x_{i}, x_{i_{5}}, \ldots, x_{i_{k+1}},\left[x_{i_{4}}, x_{q}\right]\right]\right.}\right) \\
& +\sum_{l=5}^{k+1} \delta_{i_{l}, p} \underline{\left(x_{i}^{*} \otimes\left[x_{i_{1}}, x_{i_{2}}, x_{i}, x_{i_{4}}, \ldots, x_{i_{l-1}},\left[x_{i_{l}}, x_{q}\right], x_{i_{l+1}}, \ldots, x_{i_{k+1}}\right]\right.} \\
& \quad-\delta_{i_{i, p}}\left(\frac{x_{i}^{*} \otimes\left[x_{i_{1}}, x_{i_{2}}, x_{i}, x_{i_{4}}, \ldots, x_{i_{k+1}}, x_{q}\right]-x_{i}^{*} \otimes\left[x_{i_{1}}, x_{i_{2}}, x_{i}, x_{i_{5}}, \ldots, x_{i_{k+1}}, x_{i_{4}}, x_{q}\right]}{(4)}\right) \\
& -\delta_{i, q}\left(x_{p}^{*} \otimes\left[x_{p},\left[x_{i_{1}}, x_{i_{2}}, x_{i}, x_{i_{4}}, \ldots, x_{i_{k+1}}\right]\right]-x_{p}^{*} \otimes\left[x_{p},\left[x_{i_{1}}, x_{i_{2}}, x_{i}, x_{i_{5}}, \ldots, x_{i_{k+1}}, x_{i_{4}}\right]\right]\right)
\end{aligned}
$$

we see (1) $\equiv \cdots \equiv$ (4) $\equiv 0$ by (77) and (2) of Lemma 5.1. Furthermore, if $q \neq i$, $\left[(\mathbf{A 6}), \tau_{1}\left(K_{p q}\right)\right] \equiv 0$ since all terms other than (1),.., (4) are of type (A3). Hence, we consider the case where $q=i$. In this case, $p \neq i$.

If $p \neq i_{1}, i_{2}$, it is clear $\left[(\mathbf{A 6}), \tau_{1}\left(K_{p q}\right)\right] \equiv 0$ by (A3). Suppose $p=i_{1}$. Then,

$$
\begin{aligned}
& {\left[(\mathbf{A 6}), \tau_{1}\left(K_{p q}\right)\right]} \\
& \equiv-x_{i}^{*} \otimes\left[x_{i}, x_{i_{1}}, x_{i_{2}}, x_{i}, x_{i_{4}}, \ldots, x_{i_{k+1}}\right]+x_{i}^{*} \otimes\left[x_{i}, x_{i_{1}}, x_{i_{2}}, x_{i}, x_{i_{5}}, \ldots, x_{i_{k+1}}, x_{i_{4}}\right] \\
& \quad+x_{i_{1}}^{*} \otimes\left[x_{i_{1}}, x_{i_{2}}, x_{i}, x_{i_{4}}, \ldots, x_{i_{k+1}}, x_{i_{1}}\right]-x_{i_{1}}^{*} \otimes\left[x_{i_{1}}, x_{i_{2}}, x_{i}, x_{i_{5}}, \ldots, x_{i_{k+1}}, x_{i_{4}}, x_{i_{1}}\right] \\
& \equiv 0
\end{aligned}
$$

by (A4). Similarly, we see $\left[(\mathbf{A} \mathbf{6}), \tau_{1}\left(K_{p q}\right)\right] \equiv 0$ for $p=i_{2}$. Furthermore, by an argument similar to the above, we verify that $\left[(\mathbf{A} \mathbf{6}), \tau_{1}\left(K_{p q r}\right)\right],\left[(\mathbf{A} 7), \tau_{1}\left(K_{p q}\right)\right]$ and $\left[(\mathbf{A} 7), \tau_{1}\left(K_{p q r}\right)\right] \in V_{n}(k+1)$. 
Step V. $\left[(\mathbf{A} 5), \tau_{1}\left(K_{p q}\right)\right]$.

In

$$
\begin{aligned}
& {\left[(\mathbf{A 5}), \tau_{1}\left(K_{p q}\right)\right]} \\
& =\delta_{i, p}\left(\underline{x_{i}^{*} \otimes\left[x_{i}, x_{q}, x_{i_{2}}, \ldots, x_{i_{k+1}}\right]_{(1)}}-\underline{\left.x_{i}^{*} \otimes\left[x_{i}, x_{q}, x_{i_{3}}, \ldots, x_{i_{k+1}}, x_{i_{2}}\right]_{(2)}\right)}\right. \\
& +\delta_{i_{2}, p}\left(x_{i}^{*} \otimes\left[x_{i},\left[x_{i_{2}}, x_{q}\right], x_{i_{3}}, \ldots, x_{i_{k+1}}\right]-x_{i}^{*} \otimes\left[x_{i}, x_{i_{3}}, \ldots, x_{i_{k+1}},\left[x_{i_{2}}, x_{q}\right]\right]\right) \\
& +\delta_{i_{3}, p}\left(x_{i}^{*} \otimes\left[x_{i}, x_{i_{2}},\left[x_{i_{3}}, x_{q}\right], x_{i_{4}}, \ldots, x_{i_{k+1}}\right]\right. \\
& \left.-x_{i}^{*} \otimes\left[x_{i},\left[x_{i_{3}}, x_{q}\right], x_{i_{4}}, \ldots, x_{i_{k+1}}, x_{i_{2}}\right]\right) \\
& +\delta_{i_{4}, p}\left(x_{i}^{*} \otimes\left[x_{i}, x_{i_{2}}, x_{i_{3}},\left[x_{i_{4}}, x_{q}\right], \ldots, x_{i_{k+1}}\right]\right. \\
& \left.-x_{i}^{*} \otimes\left[x_{i}, x_{i_{3}},\left[x_{i_{4}}, x_{q}\right], x_{i_{5}}, \ldots, x_{i_{k+1}}, x_{i_{2}}\right]\right) \\
& +\sum_{l=5}^{k+1} \delta_{i_{l}, p}\left(\underline{x_{i}^{*} \otimes\left[x_{i}, x_{i_{2}}, \ldots, x_{i_{l-1}},\left[x_{i_{l}}, x_{q}\right], x_{i_{l+1}}, \ldots, x_{i_{k+1}}\right]}\right. \text { (3) } \\
& \underline{\left.-x_{i}^{*} \otimes\left[x_{i}, x_{i_{3}}, \ldots, x_{i_{l-1}},\left[x_{i_{l}}, x_{q}\right], x_{i_{l+1}}, \ldots, x_{i_{k+1}}, x_{i_{2}}\right]\right)}(3) \\
& +\delta_{i, p} \underline{\left(-x_{i}^{*} \otimes\left[x_{i}, x_{i_{2}}, \ldots, x_{i_{k+1}}, x_{q}\right]_{(1)}\right.}+\underline{\left.x_{i}^{*} \otimes\left[x_{i}, x_{i_{3}}, \ldots, x_{i_{k+1}}, x_{i_{2}}, x_{q}\right]_{(2)}\right)} \\
& +\delta_{i, q}\left(-x_{p}^{*} \otimes\left[x_{p},\left[x_{i}, x_{i_{2}}, \ldots, x_{i_{k+1}}\right]\right]+x_{p}^{*} \otimes\left[x_{p},\left[x_{i}, x_{i_{3}}, \ldots, x_{i_{k+1}}, x_{i_{2}}\right]\right]\right) \text {, }
\end{aligned}
$$

(1) $\equiv(2) \equiv(3) \equiv 0$ by (7) and (A5). Furthermore, if $q \neq i$, we see $\left[(\mathbf{A 5}), \tau_{1}\left(K_{p q}\right)\right] \equiv$ 0 similarly. Hence it suffices to consider the case where $q=i$. In this case, $p \neq i$. Then using (7) and (A5), we see

$$
\begin{aligned}
& {\left[(\mathbf{A 5}), \tau_{1}\left(K_{p q}\right)\right]} \\
& \equiv \delta_{i_{2}, p}\left(x_{i}^{*} \otimes\left[x_{i}, x_{i_{2}}, x_{i}, x_{i_{3}}, \ldots, x_{i_{k+1}}\right]-x_{i}^{*} \otimes\left[x_{i}, x_{i_{3}}, \ldots, x_{i_{k+1}}, x_{i_{2}}, x_{i}\right]\right. \\
& \left.+x_{i}^{*} \otimes\left[x_{i}, x_{i_{3}}, \ldots, x_{i_{k+1}}, x_{i}, x_{i_{2}}\right]\right) \\
& +\delta_{i_{3}, p}\left(x_{i}^{*} \otimes\left[x_{i}, x_{i_{2}}, x_{i_{3}}, x_{i}, x_{i_{4}}, \ldots, x_{i_{k+1}}\right]-x_{i}^{*} \otimes\left[x_{i}, x_{i_{2}}, x_{i}, x_{i_{3}}, x_{i_{4}}, \ldots, x_{i_{k+1}}\right]\right. \\
& \left.-x_{i}^{*} \otimes\left[x_{i}, x_{i_{3}}, x_{i}, x_{i_{4}}, \ldots, x_{i_{k+1}}, x_{i_{2}}\right]\right) \\
& -\delta_{i_{4}, p}\left(x_{i}^{*} \otimes\left[x_{i}, x_{i_{2}}, x_{i_{3}}, x_{i}, x_{i_{4}}, \ldots, x_{i_{k+1}}\right]-x_{i}^{*} \otimes\left[x_{i}, x_{i_{3}}, x_{i}, x_{i_{4}}, x_{i_{5}}, \ldots, x_{i_{k+1}}, x_{i_{2}}\right]\right) \\
& +x_{p}^{*} \otimes\left[x_{i}, x_{i_{2}}, \ldots, x_{i_{k+1}}, x_{p}\right]-x_{p}^{*} \otimes\left[x_{i}, x_{i_{3}}, \ldots, x_{i_{k+1}}, x_{i_{2}}, x_{p}\right] .
\end{aligned}
$$

Since $n \geq 6$, there exist some $1 \leq j \leq n$ such that $j \neq i, i_{2}, i_{3}, i_{4}$. We fix it.

Case I. $i_{2}, i_{3}$ and $i_{4}$ are distinct.

If $i_{2}, i_{3}$ and $i_{4}$ are distinct, using (A3), we have

$$
\begin{aligned}
& {\left[(\mathbf{A} \mathbf{5}), \tau_{1}\left(K_{p q}\right)\right]} \\
& \begin{array}{l}
\equiv \delta_{i_{2}, p}\left(x_{i}^{*} \otimes\left[x_{i}, x_{i_{2}}, x_{i}, x_{i_{3}}, \ldots, x_{i_{k+1}}\right]-x_{i}^{*} \otimes\left[x_{i}, x_{i_{3}}, \ldots, x_{i_{k+1}}, x_{i_{2}}, x_{i}\right]\right. \\
\left.\quad+x_{i}^{*} \otimes\left[x_{i}, x_{i_{3}}, \ldots, x_{i_{k+1}}, x_{i}, x_{i_{2}}\right]+x_{i_{2}}^{*} \otimes\left[x_{i}, x_{i_{2}}, x_{i_{3}}, \ldots, x_{i_{k+1}}, x_{i_{2}}\right]\right) \\
\quad+\delta_{i_{3}, p}\left(x_{i}^{*} \otimes\left[x_{i}, x_{i_{2}}, x_{i_{3}}, x_{i}, x_{i_{4}}, \ldots, x_{i_{k+1}}\right]-x_{i}^{*} \otimes\left[x_{i}, x_{i_{2}}, x_{i}, x_{i_{3}}, x_{i_{4}}, \ldots, x_{i_{k+1}}\right]\right. \\
\quad-x_{i}^{*} \otimes\left[x_{i}, x_{i_{3}}, x_{i}, x_{i_{4}}, \ldots, x_{i_{k+1}}, x_{i_{2}}\right]+x_{i_{3}}^{*} \otimes\left[x_{i}, x_{i_{2}}, x_{i_{3}}, x_{i_{4}}, \ldots, x_{i_{k+1}}, x_{i_{3}}\right] \\
\left.\quad-x_{i_{3}}^{*} \otimes\left[x_{i}, x_{i_{3}}, x_{i_{4}}, \ldots, x_{i_{k+1}}, x_{i_{2}}, x_{i_{3}}\right]\right) \\
\quad-\delta_{i_{4}, p}\left(x_{i}^{*} \otimes\left[x_{i}, x_{i_{2}}, x_{i_{3}}, x_{i}, x_{i_{4}}, \ldots, x_{i_{k+1}}\right]-x_{i}^{*} \otimes\left[x_{i}, x_{i_{3}}, x_{i}, x_{i_{4}}, x_{i_{5}}, \ldots, x_{i_{k+1}}, x_{i_{2}}\right]\right. \\
\left.\quad+x_{i_{4}}^{*} \otimes\left[x_{i}, x_{i_{3}}, x_{i_{4}}, \ldots, x_{i_{k+1}}, x_{i_{2}}, x_{i_{4}}\right]\right) .
\end{array}
\end{aligned}
$$


Then from (A8) and (3) of Lemma 5.1 the $\delta_{i_{2}, p}$-part is equal to

$$
\begin{aligned}
x_{j}^{*} \otimes & {\left[x_{j}, x_{i_{2}}, x_{i}, x_{i_{3}}, \ldots, x_{i_{k+1}}\right]+x_{j}^{*} \otimes\left[x_{i}, x_{i_{2}}, x_{j}, x_{i_{3}}, \ldots, x_{i_{k+1}}\right] } \\
& -x_{j}^{*} \otimes\left[x_{j}, x_{i_{3}}, \ldots, x_{i_{k+1}}, x_{i_{2}}, x_{i}\right]+x_{j}^{*} \otimes\left[x_{j}, x_{i_{3}}, \ldots, x_{i_{k+1}}, x_{i}, x_{i_{2}}\right] \\
& -x_{j}^{*} \otimes\left[x_{j}, x_{i}, x_{i_{3}}, \ldots, x_{i_{k+1}}, x_{i_{2}}\right] .
\end{aligned}
$$

Hence, by (A5), we obtain that the $\delta_{i_{2}, p}$-part is equal to zero modulo $V_{n}(k+1)$. Similarly, we see that the $\delta_{i_{3}, p}$-part and the $\delta_{i_{4}, p}$-part of the equation above are equal to zero modulo $V_{n}(k+1)$. Therefore we obtain $\left[(\mathbf{A} 5), \tau_{1}\left(K_{p q}\right)\right] \equiv 0$.

Case II. $i_{2}=i_{3} \neq i_{4}$.

If $i_{2}=i_{3}=m$ and $i_{4} \neq m$, using (A3), we have

$$
\begin{aligned}
& {\left[(\mathbf{A 5}), \tau_{1}\left(K_{p q}\right)\right]} \\
& \equiv \delta_{m, p} \underline{\left(x_{i}^{*} \otimes\left[x_{i}, x_{m}, x_{i}, x_{m}, x_{i_{4}}, \ldots, x_{i_{k+1}}\right]_{(4)}\right.}-x_{i}^{*} \otimes\left[x_{i}, x_{m}, x_{i_{4}}, \ldots, x_{i_{k+1}}, x_{m}, x_{i}\right] \\
& \quad+x_{i}^{*} \otimes\left[x_{i}, x_{m}, x_{i_{4}}, \ldots, x_{i_{k+1}}, x_{i}, x_{m}\right]+x_{i}^{*} \otimes\left[x_{i}, x_{m}, x_{m}, x_{i}, x_{i_{4}}, \ldots, x_{i_{k+1}}\right] \\
& \frac{-x_{i}^{*} \otimes\left[x_{i}, x_{m}, x_{i}, x_{m}, x_{i_{4}}, \ldots, x_{i_{k+1}}\right]_{(4)}-x_{i}^{*} \otimes\left[x_{i}, x_{m}, x_{i}, x_{i_{4}}, \ldots, x_{i_{k+1}}, x_{m}\right]}{\left.-x_{m}^{*} \otimes\left[x_{m}, x_{i}, x_{m}, x_{i_{4}}, \ldots, x_{i_{k+1}}, x_{m}\right]-x_{m}^{*} \otimes\left[x_{i}, x_{m}, x_{i_{4}}, \ldots, x_{i_{k+1}}, x_{m}, x_{m}\right]\right)} \\
& -\delta_{i_{4}, p}\left(x_{i}^{*} \otimes\left[x_{i}, x_{m}, x_{m}, x_{i}, x_{i_{4}}, \ldots, x_{i_{k+1}}\right]-x_{i}^{*} \otimes\left[x_{i}, x_{m}, x_{i}, x_{i_{4}}, \ldots, x_{i_{k+1}}, x_{m}\right]\right. \\
& \left.\quad+x_{i_{4}}^{*} \otimes\left[x_{i}, x_{m}, x_{i_{4}}, \ldots, x_{i_{k+1}}, x_{m}, x_{i_{4}}\right]\right) .
\end{aligned}
$$

In the $\delta_{m, p}$-part, (4) $=0$. From (A8) and (3) of Lemma 5.1, the other terms are equal to

$$
\begin{aligned}
& \left.\underline{-x_{j}^{*} \otimes\left[x_{j}, x_{m}, x_{i_{4}}, \ldots, x_{i_{k+1}}, x_{m}, x_{i}\right]+x_{j}^{*} \otimes\left[x_{j}, x_{m}, x_{i_{4}}, \ldots, x_{i_{k+1}}, x_{i}, x_{m}\right]}\right]_{6}
\end{aligned}
$$

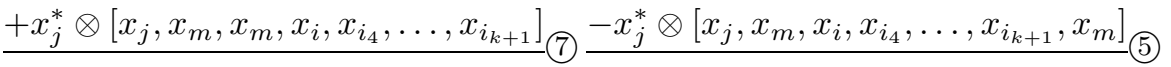

$$
\begin{aligned}
& -x_{j}^{*} \otimes\left[x_{i}, x_{m}, x_{j}, x_{i_{4}}, \ldots, x_{i_{k+1}}, x_{m}\right] \underline{-x_{j}^{*} \otimes\left[x_{j}, x_{i}, x_{m}, x_{i_{4}}, \ldots, x_{i_{k+1}}, x_{m}\right]_{7}}(7) \\
& -x_{j}^{*} \otimes\left[x_{m}, x_{i}, x_{j}, x_{i_{4}}, \ldots, x_{i_{k+1}}, x_{m}\right] \underline{+x_{j}^{*} \otimes\left[x_{j}, x_{i}, x_{i_{4}}, \ldots, x_{i_{k+1}}, x_{m}, x_{m}\right]_{(5)}}
\end{aligned}
$$

modulo $V_{n}(k+1)$. Then (5) $\equiv 0$ by (A5), and

$$
\begin{aligned}
(6) & \equiv-x_{j}^{*} \otimes\left[x_{j}, x_{m}, x_{i}, x_{m}, x_{i_{4}}, \ldots, x_{i_{k+1}}\right]+x_{j}^{*} \otimes\left[x_{j}, x_{i}, x_{m}, x_{m}, x_{i_{4}}, \ldots, x_{i_{k+1}}\right] \\
& \equiv x_{j}^{*} \otimes\left[x_{m}, x_{i}, x_{j}, x_{m}, x_{i_{4}}, \ldots, x_{i_{k+1}}\right]
\end{aligned}
$$

by (A5) and (1) of Lemma 5.1. Similarly,

$$
\text { (7) } \equiv x_{j}^{*} \otimes\left[x_{i}, x_{m}, x_{j}, x_{i_{4}}, \ldots, x_{i_{k+1}}, x_{m}\right] .
$$

Hence, using (2) of Lemma 5.1, we see that the $\delta_{m, p}$-part $\equiv 0$. Similarly, we can show the $\delta_{i_{4}, p}$-part $\equiv 0$, and hence,

$$
\left[(\mathbf{A 5}), \tau_{1}\left(K_{p q}\right)\right] \equiv 0 .
$$

By an argument similar to the above, we show $\left[(\mathbf{A 5}), \tau_{1}\left(K_{p q}\right)\right] \equiv 0$ for the other cases $i_{2}=i_{4} \neq i_{3}, i_{3}=i_{4} \neq i_{2}$ and $i_{2}=i_{3}=i_{4}$. Furthermore we obtain $\left[(\mathbf{A 5}), \tau_{1}\left(K_{p q r}\right)\right], \quad\left[(\mathbf{A} 4), \tau_{1}\left(K_{p q}\right)\right], \quad\left[(\mathbf{A} 4), \tau_{1}\left(K_{p q r}\right)\right], \quad\left[(\mathbf{A} 8), \tau_{1}\left(K_{p q}\right)\right] \quad$ and $\left[(\mathbf{A} \mathbf{8}), \tau_{1}\left(K_{p q r}\right)\right] \in V_{n}(k+1)$. We leave it to the reader for exercises. This completes the proof of Theorem 5.1 
5.2. Contractions and trace maps. The main purpose of this subsection is to detect the module $S^{k} H_{\mathbf{Q}}$ and $H_{\mathbf{Q}}^{\left[k-2,1^{2}\right]}$ in the cokernel $\left(\tau_{k, N}^{\prime}\right)_{\mathbf{Q}}$ using trace maps. For $k \geq 1$ and $1 \leq l \leq k+1$, let $\varphi_{l}^{k}: H^{*} \otimes_{\mathbf{z}} H^{\otimes(k+1)} \rightarrow H^{\otimes k}$ be the contraction map defined by

$$
x_{i}^{*} \otimes x_{j_{1}} \otimes \cdots \otimes x_{j_{k+1}} \mapsto x_{i}^{*}\left(x_{j_{l}}\right) \cdot x_{j_{1}} \otimes \cdots \otimes x_{j_{l-1}} \otimes x_{j_{l+1}} \otimes \cdots \otimes x_{j_{k+1}} .
$$

For the natural embedding $\iota_{n}^{k+1}: \mathcal{L}_{n}(k+1) \rightarrow H^{\otimes(k+1)}$, we obtain a $\operatorname{GL}(n, \mathbf{Z})$ equivariant homomorphism

$$
\Phi_{l}^{k}=\varphi_{l}^{k} \circ\left(i d_{H^{*}} \otimes \iota_{n}^{k+1}\right): H^{*} \otimes \mathbf{Z} \mathcal{L}_{n}(k+1) \rightarrow H^{\otimes k} .
$$

We also call $\Phi_{l}^{k}$ a contraction map.

For any $x_{i}^{*} \otimes\left[x_{i_{1}}, \ldots, x_{i_{k+1}}\right] \in H^{*} \otimes \mathbf{z} H^{\otimes k}$, and $1 \leq m \leq k+1$, let

$$
\Phi_{l, m}^{k}\left(x_{i}^{*} \otimes\left[x_{i_{1}}, \ldots, x_{i_{k+1}}\right]\right)
$$

denote the element obtained by the contraction of $x_{i}^{*}$ with the only element $x_{j_{m}}$. For example,

$$
\begin{aligned}
& \Phi_{1,2}^{3}\left(x_{i}^{*} \otimes\left[x_{i_{1}}, x_{i_{2}}, x_{i_{3}}\right]\right) \\
& \quad=\Phi_{1,2}^{3}\left(x _ { i } ^ { * } \otimes \left(x_{i_{1}} \otimes x_{i_{2}} \otimes x_{i_{3}}-x_{i_{2}} \otimes x_{i_{1}} \otimes x_{i_{3}}-x_{i_{3}} \otimes x_{i_{1}} \otimes x_{i_{2}}\right.\right. \\
& \left.\left.\quad \quad+x_{i_{3}} \otimes x_{i_{2}} \otimes x_{i_{1}}\right)\right) \\
& \quad=-\delta_{i i_{2}} x_{i_{1}} \otimes x_{i_{3}}, \\
& \Phi_{1,3}^{3}\left(x_{i}^{*} \otimes\left[x_{i_{1}}, x_{i_{2}}, x_{i_{3}}\right]\right)=-\delta_{i i_{3}} x_{i_{1}} \otimes x_{i_{2}}+\delta_{i i_{3}} x_{i_{2}} \otimes x_{i_{1}} .
\end{aligned}
$$

Then we have

$$
\Phi_{l}^{k}\left(x_{i}^{*} \otimes\left[x_{i_{1}}, \ldots, x_{i_{k+1}}\right]\right)=\sum_{m=1}^{k+1} \Phi_{l, m}^{k}\left(x_{i}^{*} \otimes\left[x_{i_{1}}, \ldots, x_{i_{k+1}}\right]\right) .
$$

Here we make sure that $H^{*} \otimes \mathbf{z} \mathcal{L}_{n}^{N}(k)$ is written as a quotient module of $H^{*} \otimes \mathbf{z}$ $\mathcal{L}_{n}(k)$, which is used to define the trace maps later. For each $k \geq 5$, if we set $Q_{n}(k):=\left(\Gamma_{n}(k) \cap K\right) \Gamma_{n}(k+1) / \Gamma_{n}(k+1)$, we have an exact sequence

$$
0 \rightarrow Q_{n}(k) \rightarrow \mathcal{L}_{n}(k) \rightarrow \mathcal{L}_{n}^{N}(k) \rightarrow 0
$$

of $\mathrm{GL}(n, \mathbf{Z})$-equivariant free abelian groups. This induces an exact sequence

$$
0 \rightarrow H^{*} \otimes_{\mathbf{Z}} Q_{n}(k) \rightarrow H^{*} \otimes_{\mathbf{Z}} \mathcal{L}_{n}(k) \rightarrow H^{*} \otimes_{\mathbf{Z}} \mathcal{L}_{n}^{N}(k) \rightarrow 0 .
$$

Therefore we can regard $H^{*} \otimes_{\mathbf{z}} \mathcal{L}_{n}^{N}(k)$ as a quotient module of $H^{*} \otimes_{\mathbf{z}} \mathcal{L}_{n}(k)$ by $H^{*} \otimes_{\mathbf{z}} Q_{n}(k)$. Since the basic commutators of types

$$
\left[x_{i_{1}}, \ldots, x_{i_{k}}\right] \text { and }\left[x_{i_{1}}, \ldots, x_{i_{k-2}},\left[x_{i_{k-1}}, x_{i_{k}}\right]\right]
$$

form a basis of the free abelian group $\mathcal{L}_{n}^{N}(k)$ by Theorem 4.1, those of type

$$
\left[c_{1}, c_{2}\right] \text { for } \operatorname{wt}\left(c_{1}\right), \operatorname{wt}\left(c_{2}\right) \geq 3
$$

and

$$
\left[c_{1}, c_{2}, c_{3}\right] \text { for } \quad \operatorname{wt}\left(c_{1}\right), \operatorname{wt}\left(c_{2}\right), \operatorname{wt}\left(c_{3}\right) \geq 2
$$

form a basis of $Q_{n}(k)$. 
5.2.1. The Morita trace. Here we recall the Morita trace map. Let

$$
\operatorname{Tr}_{[k]}: H^{*} \otimes \mathbf{z} \mathcal{L}_{n}(k+1) \rightarrow S^{k} H
$$

be the composition map of the contraction $\Phi_{1}^{k}$ and the natural projection $f_{[k]}$ : $H^{\otimes k} \rightarrow S^{k} H$ defined by

$$
f_{[k]}\left(x_{i_{1}} \otimes \cdots \otimes x_{i_{k}}\right)=x_{i_{1}} \cdots x_{i_{k}} .
$$

The Morita trace was introduced with remarkable pioneer works by Shigeyuki Morita who showed that $\operatorname{Tr}_{[k]}$ is surjective and vanishes on the image of the Johnson homomorphism $\tau_{k}$ for $n \geq 3$ and $k \geq 2$. This shows that $S^{k} H_{\mathbf{Q}}$ appears in the irreducible decomposition of $\operatorname{Coker}\left(\tau_{k, \mathbf{Q}}\right)$ and $\operatorname{Coker}\left(\tau_{k, \mathbf{Q}}^{\prime}\right)$ as a $\mathrm{GL}(n, \mathbf{Z})$-module. We call $S^{k} H_{\mathbf{Q}}$ the Morita obstruction.

Let $c=\left[c_{1}, c_{2}\right] \in \Gamma_{n}(k+1)$ be a basic commutator of weight $k+1$ such that $\operatorname{wt}\left(c_{1}\right), \operatorname{wt}\left(c_{2}\right) \geq 2$. Then for any $1 \leq i \leq n$,

$$
\Phi_{1}^{k}\left(x_{i}^{*} \otimes c\right)=\Phi_{1}^{k}\left(x_{i}^{*} \otimes c_{1}\right) \otimes c_{2}-\Phi_{1}^{k}\left(x_{i}^{*} \otimes c_{2}\right) \otimes c_{1} \in H^{\otimes k} .
$$

Hence $\operatorname{Tr}_{[k]}\left(x_{i}^{*} \otimes c\right)=0$. This shows that $\operatorname{Tr}_{[k]}$ factors through $H^{*} \otimes \mathbf{z} \mathcal{L}_{n}^{N}(k)$. Therefore we see that the Morita obstruction $S^{k} H_{\mathbf{Q}}$ also appears in $\operatorname{Coker}\left(\left(\tau_{k, N}^{\prime}\right)_{\mathbf{Q}}\right)$.

5.2.2. Trace map for $H^{\left[k-2,1^{2}\right]}$. Next we detect $H_{\mathbf{Q}}^{\left[k-2,1^{2}\right]}$ in the cokernel $\left(\tau_{k, N}^{\prime}\right)_{\mathbf{Q}}$. Let $\mu: H^{\otimes k} \rightarrow \Lambda^{3} H \otimes \mathbf{z} S^{k-3} H$ be a homomorphism defined by

$$
x_{i_{1}} \otimes \cdots \otimes x_{i_{k}} \mapsto\left(x_{i_{1}} \wedge x_{i_{2}} \wedge x_{i_{3}}\right) \otimes x_{i_{4}} \cdots x_{i_{k}} .
$$

Since $H^{\left[k-2,1^{2}\right]}$ is considered as a quotient module of $\Lambda^{3} H \otimes_{\mathbf{z}} S^{k-3} H$ (see [6]), we have a natural projection $\nu: \Lambda^{3} H \otimes \mathbf{z} S^{k-3} H \rightarrow H^{\left[k-2,1^{2}\right]}$. Let

$$
\operatorname{Tr}_{\left[k-2,1^{2}\right]}: H^{*} \otimes \mathbf{z} \mathcal{L}_{n}(k+1) \rightarrow H^{\left[k-2,1^{2}\right]}
$$

be the composition of $\Phi_{4}^{k}$ and $f_{\left[k-2,1^{2}\right]}:=\nu \circ \mu$. The map $\operatorname{Tr}_{\left[k-2,1^{2}\right]}$ is a $\operatorname{GL}(n, \mathbf{Z})$ equivariant homomorphism. We call it the trace map for $H^{\left[k-2,1^{2}\right]}$. In the following, we show

Theorem 5.2. For $n \geq 3$ and $k \geq 3$,

(1) $\operatorname{Tr}_{\left[k-2,1^{2}\right]}^{\mathbf{Q}}$ is surjective,

(2) $\operatorname{Tr}_{\left[k-2,1^{2}\right]} \circ \tau_{k}^{\prime} \equiv 0$.

To show part (2) of the theorem above, it suffices to show that $\operatorname{Tr}_{\left[k-2,1^{2}\right]}$ vanishes on $(\mathbf{A} 1), \ldots,(\mathbf{A} 8)$ in Theorem 5.1 .

Lemma 5.2. For $k \geq 5$,

(1) $\operatorname{Tr}_{\left[k-2,1^{2}\right]}\left(x_{i}^{*} \otimes[A, B]\right)=0$ for $\operatorname{wt}(A), \operatorname{wt}(B) \geq 3$,

(2) $\operatorname{Tr}_{\left[k-2,1^{2}\right]}\left(x_{i}^{*} \otimes[A, B, C]\right)=0$ for $\operatorname{wt}(A), \operatorname{wt}(B), \operatorname{wt}(C) \geq 2$.

Proof. For part (1), we may assume $\operatorname{wt}(A) \geq \operatorname{wt}(B)$. If $\operatorname{wt}(B)=4$, we have

$$
\Phi_{4}^{k}\left(x_{i}^{*} \otimes[A, B]\right)=\Phi_{4}^{k}\left(x_{i}^{*} \otimes A\right) \otimes B-\Phi_{4}^{k}\left(x_{i}^{*} \otimes B\right) \otimes A .
$$

If $\operatorname{wt}(A) \geq 4$ and $\operatorname{wt}(B)=3$,

$$
\Phi_{4}^{k}\left(x_{i}^{*} \otimes[A, B]\right)=\Phi_{4}^{k}\left(x_{i}^{*} \otimes A\right) \otimes B-B \otimes \Phi_{1}^{k-3}\left(x_{i}^{*} \otimes A\right) .
$$

If $\operatorname{wt}(A)=\operatorname{wt}(B)=3$,

$$
\Phi_{4}^{k}\left(x_{i}^{*} \otimes[A, B]\right)=A \otimes \Phi_{1}^{k-3}\left(x_{i}^{*} \otimes B\right)-B \otimes \Phi_{1}^{k-3}\left(x_{i}^{*} \otimes A\right) .
$$


Hence, we obtain $\operatorname{Tr}_{\left[k-2,1^{2}\right]}\left(x_{i}^{*} \otimes[A, B]\right)=0$ for any case. Similarly, we see part (2). This completes the proof of Lemma 5.2 .

From this lemma, we verify that $\operatorname{Tr}_{\left[k-2,1^{2}\right]}$ vanishes on (A1) and (A2).

Lemma 5.3. For $k \geq 3$ and $4 \leq m \leq k+1$,

$$
f_{\left[k-2,1^{2}\right]} \circ \Phi_{4, m}^{k}\left(x_{i}^{*} \otimes\left[x_{i_{1}}, x_{i_{2}}, \ldots, x_{i_{k+1}}\right]\right)=0 .
$$

Proof. Since the element $\left[x_{i_{1}}, x_{i_{2}}, \ldots, x_{i_{k+1}}\right]$ in $H^{\otimes k}$ is written as a sum of elements of types

$$
A \otimes\left[x_{i_{1}}, \ldots, x_{i_{m-1}}\right] \otimes x_{i_{m}} \otimes B \quad \text { or } \quad A \otimes x_{i_{m}} \otimes\left[x_{i_{1}}, \ldots, x_{i_{m-1}}\right] \otimes B,
$$

$\Phi_{4, m}^{k}\left(x_{i}^{*} \otimes\left[x_{i_{1}}, x_{i_{2}}, \ldots, x_{i_{k+1}}\right]\right)$ is a sum of elements of types

$$
\delta_{i i_{4}}\left[x_{i_{1}}, x_{i_{2}}, x_{i_{3}}\right] \otimes B
$$

or

$$
\delta_{i i_{m}} A \otimes\left[x_{i_{1}}, \ldots, x_{i_{m-1}}\right] \otimes B \quad \text { for } \quad A \in H^{\otimes 3} .
$$

Considering the image of $f_{\left[k-2,1^{2}\right]}$, we obtain the required result. This completes the proof of Lemma 5.3 .

Corollary 5.1. For $k \geq 3$,

$$
\operatorname{Tr}_{\left[k-2,1^{2}\right]}\left(x_{i}^{*} \otimes\left[x_{i_{1}}, x_{i_{2}}, \ldots, x_{i_{k+1}}\right]\right)=0
$$

if $i \neq i_{1}, i_{2}, i_{3}$. That is, $\operatorname{Tr}_{\left[k-2,1^{2}\right]}$ vanishes on (A3).

Proof. Since

$$
\begin{aligned}
\operatorname{Tr}_{\left[k-2,1^{2}\right]}\left(x_{i}^{*}\right. & \left.\otimes\left[x_{i_{1}}, x_{i_{2}}, \ldots, x_{i_{k+1}}\right]\right) \\
& =\sum_{m=1}^{k+1} f_{\left[k-2,1^{2}\right]} \circ \Phi_{4, m}^{k}\left(x_{i}^{*} \otimes\left[x_{i_{1}}, x_{i_{2}}, \ldots, x_{i_{k+1}}\right]\right),
\end{aligned}
$$

we immediately obtain the required result from Lemma 5.3 .

Lemma 5.4. For $k \geq 3$, and $i \neq i_{2}, i_{3}$,

$$
\begin{aligned}
& \operatorname{Tr}_{\left[k-2,1^{2}\right]}\left(x_{i}^{*} \otimes\left[x_{i}, x_{i_{2}}, x_{i_{3}}, \ldots, x_{i_{k+1}}\right]\right) \\
& \quad=-\sum_{2 \leq l_{3}<l_{2}<l_{1} \leq k+1}\left(x_{i_{l_{1}}} \wedge x_{i_{l_{2}}} \wedge x_{i_{l_{3}}}\right) \otimes x_{i_{2}} \cdots \check{x_{i_{3}}} \cdots \check{i_{i_{2}}} \cdots x_{i_{l_{1}}}^{\check{\nu}} \cdots x_{i_{k+1}} .
\end{aligned}
$$

Here $\check{y}$ means removing $y$ in the product.

Proof. From Lemma 5.3 and $i \neq i_{2}, i_{3}$, we see

$$
\begin{aligned}
\operatorname{Tr}_{\left[k-2,1^{2}\right]}\left(x_{i}^{*}\right. & \left.\otimes\left[x_{i}, x_{i_{2}}, x_{i_{3}}, \ldots, x_{i_{k+1}}\right]\right) \\
& =f_{\left[k-2,1^{2}\right]} \circ \Phi_{4,1}^{k}\left(x_{i}^{*} \otimes\left[x_{i}, x_{i_{2}}, x_{i_{3}}, \ldots, x_{i_{k+1}}\right]\right) .
\end{aligned}
$$

On the other hand, in general, if we write $\left[x_{j_{1}}, x_{j_{2}}, x_{j_{3}}, \ldots, x_{j_{k+1}}\right] \in H^{\otimes k+1}$ as a sum of elements $x_{j_{1}^{\prime}} \otimes \cdots \otimes x_{j_{k+1}^{\prime}}$, the sum of the elements such that $j_{4}^{\prime}=j_{1}$ is given by

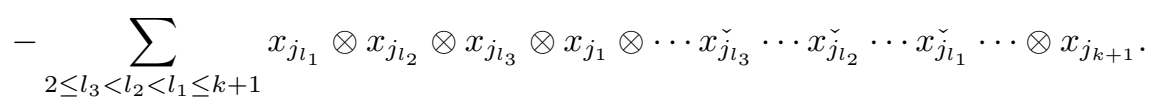

Hence we obtain the required result. This completes the proof of Lemma 5.4. 
This lemma induces

Corollary 5.2. For $k \geq 3$, we have:

(1) For $i \neq i_{2}, i_{3}, \quad j \neq i_{3}, i_{4}$ and $i \neq j$,

$\operatorname{Tr}_{\left[k-2,1^{2}\right]}\left(x_{i}^{*} \otimes\left[x_{i}, x_{i_{2}}, x_{i_{3}}, \ldots, x_{i_{k+1}}\right]-x_{j}^{*} \otimes\left[x_{j}, x_{i_{3}}, x_{i_{4}}, \ldots, x_{i_{k+1}}, x_{i_{2}}\right]\right)=0$.

(2) For $i \neq i_{2}, i_{3}, i_{4}$,

$\operatorname{Tr}_{\left[k-2,1^{2}\right]}\left(x_{i}^{*} \otimes\left[x_{i}, x_{i_{2}}, x_{i_{3}}, \ldots, x_{i_{k+1}}\right]-x_{i}^{*} \otimes\left[x_{i}, x_{i_{3}}, x_{i_{4}}, \ldots, x_{i_{k+1}}, x_{i_{2}}\right]\right)=0$.

Hence we verify that $\operatorname{Tr}_{\left[k-2,1^{2}\right]}$ vanishes on (A4) and (A5).

Lemma 5.5. For $k \geq 3$ and $i \neq i_{1}, i_{2}$,

$$
\begin{aligned}
\operatorname{Tr}_{\left[k-2,1^{2}\right]}\left(x_{i}^{*}\right. & \left.\otimes\left[x_{i_{1}}, x_{i_{2}}, x_{i}, x_{i_{4}}, \ldots, x_{i_{k+1}}\right]\right) \\
& =-\sum_{j=4}^{k+1} 2\left(x_{i_{j}} \wedge x_{i_{1}} \wedge x_{i_{2}}\right) \otimes x_{i_{4}} \cdots \check{x_{i_{j}}} \cdots x_{i_{k+1}} .
\end{aligned}
$$

Proof. From Lemma 5.3 and $i \neq i_{1}, i_{2}$, we see

$$
\begin{aligned}
\operatorname{Tr}_{\left[k-2,1^{2}\right]}\left(x_{i}^{*}\right. & \left.\otimes\left[x_{i_{1}}, x_{i_{2}}, x_{i}, x_{i_{4}}, \ldots, x_{i_{k+1}}\right]\right) \\
& =f_{\left[k-2,1^{2}\right]} \circ \Phi_{4,3}^{k}\left(x_{i}^{*} \otimes\left[x_{i_{1}}, x_{i_{2}}, x_{i}, x_{i_{4}}, \ldots, x_{i_{k+1}}\right]\right) .
\end{aligned}
$$

In general, an element $\left[x_{j_{1}}, x_{j_{2}}, x_{j_{3}}, \ldots, x_{j_{k+1}}\right] \in H^{\otimes k+1}$ is written as a sum of elements of types

$$
A \otimes x_{j_{3}} \otimes\left[x_{j_{1}}, x_{j_{2}}\right] \otimes B \quad \text { or } \quad A \otimes\left[x_{j_{1}}, x_{j_{2}}\right] \otimes x_{j_{3}} \otimes B .
$$

Hence $\Phi_{4,3}^{k}\left(x_{i}^{*} \otimes\left[x_{i_{1}}, x_{i_{2}}, x_{i}, x_{i_{4}}, \ldots, x_{i_{k+1}}\right]\right)$ is a written as a sum of

$$
A \otimes\left[x_{i_{1}}, x_{i_{2}}\right] \otimes B
$$

for $\operatorname{wt}(A)=3$, or

$$
x_{i_{j}} \otimes\left[x_{i_{1}}, x_{i_{2}}\right] \otimes B
$$

for $4 \leq j \leq k+1$. Then $f_{\left[k-2,1^{2}\right]}\left(A \otimes\left[x_{i_{1}}, x_{i_{2}}\right] \otimes B\right)=0$ for $\operatorname{wt}(A)=3$.

On the other hand, in $\left[x_{j_{1}}, x_{j_{2}}, x_{j_{3}}, \ldots, x_{j_{k+1}}\right] \in H^{\otimes k+1}$, the sum of the elements of type $x_{j_{l}} \otimes\left[x_{j_{1}}, x_{j_{2}}\right] \otimes B$ is given by

$$
-\sum_{l=4}^{k+1} x_{j_{l}} \otimes\left[x_{j_{1}}, x_{j_{2}}\right] \otimes x_{j_{4}} \otimes \cdots \check{x_{j_{l}}} \cdots \otimes x_{j_{k+1}} .
$$

From this, we obtain Lemma 5.5

Lemma 5.4 induces

Corollary 5.3. For $k \geq 3$ and any $\gamma \in \mathfrak{S}_{k-2}$,

$\operatorname{Tr}_{\left[k-2,1^{2}\right]}\left(x_{i}^{*} \otimes\left[x_{i_{1}}, x_{i_{2}}, x_{i}, x_{j_{1}}, \ldots, x_{j_{k-2}}\right]-x_{i}^{*} \otimes\left[x_{i_{1}}, x_{i_{2}}, x_{i}, x_{j_{\gamma(1)}}, \ldots, x_{j_{\gamma(k-2)}}\right]\right)=0$.

That is, $\operatorname{Tr}_{\left[k-2,1^{2}\right]}$ vanishes on (A6) and (A7).

Furthermore, by an argument similar to that in Lemmas 5.4 and 5.5 , we obtain

Lemma 5.6. For $i, j \neq i_{2}$ and $i \neq j$,

$$
\begin{gathered}
\operatorname{Tr}_{\left[k-2,1^{2}\right]}\left(x_{i}^{*} \otimes\left[x_{i}, x_{i_{2}}, x_{i}, x_{i_{4}}, \ldots, x_{i_{k+1}}\right]-x_{j}^{*} \otimes\left[x_{j}, x_{i_{2}}, x_{i}, x_{i_{4}}, \ldots, x_{i_{k+1}}\right]\right. \\
\left.-x_{j}^{*} \otimes\left[x_{i}, x_{i_{2}}, x_{j}, x_{i_{4}}, \ldots, x_{i_{k+1}}\right]\right)=0 .
\end{gathered}
$$

Proof. We leave the calculations to the reader for exercises. 
Therefore $\operatorname{Tr}_{\left[k-2,1^{2}\right]}$ vanishes on (A8). Finally, we consider the surjectivity of $\operatorname{Tr}_{\left[k-2,1^{2}\right]}^{\mathbf{Q}}$. Since $n \geq 3$, we can choose distinct $1 \leq i, j, l \leq n$. Then from Lemma 5.5 .

$$
\begin{aligned}
\operatorname{Tr}_{\left[k-2,1^{2}\right]}^{\mathbf{Q}}\left(x_{i}^{*} \otimes\left[x_{j}, x_{l}, x_{i}, x_{i}, \ldots, x_{i}\right]\right) & =-2(k-2)\left(x_{i} \wedge x_{j} \wedge x_{l}\right) \otimes x_{i} \cdots x_{i}, \\
& \neq 0
\end{aligned}
$$

in $H_{\mathbf{Q}}^{\left[k-2,1^{2}\right]}$. Since $H_{\mathbf{Q}}^{\left[k-2,1^{2}\right]}$ is irreducible, we see that $\operatorname{Tr}_{\left[k-2,1^{2}\right]}^{\mathbf{Q}}$ is surjective. This completes the proof of Theorem 5.2. As a corollary, we obtain

Corollary 5.4. For $n \geq 3$ and $k \geq 3$,

(1) $H_{\mathbf{Q}}^{\left[k-2,1^{2}\right]} \subset \operatorname{Coker}\left(\tau_{k, \mathbf{Q}}^{\prime}\right)$,

(2) $H_{\mathbf{Q}}^{\left[k-2,1^{2}\right]} \subset \operatorname{Coker}\left(\left(\tau_{k, N}^{\prime}\right)_{\mathbf{Q}}\right)$.

Proof. Part (1) is clear. Part (2) follows from the fact that $\operatorname{Tr}_{\left[k-2,1^{2}\right]}$ factors through $H^{*} \otimes_{\mathbf{Z}} \mathcal{L}_{n}^{N}(k)$ since $\operatorname{Tr}_{\left[k-2,1^{2}\right]}$ vanishes on (A1) and (A2).

5.3. An upper bound on $\operatorname{Coker}\left(\left(\tau_{k, N}^{\prime}\right)_{\mathbf{Q}}\right)$. In this subsection, we show that $\operatorname{Coker}\left(\left(\tau_{k, N}^{\prime}\right)_{\mathbf{Q}}\right)$ is a direct sum of $S^{k} H_{\mathbf{Q}}$ and $H_{\mathbf{Q}}^{\left[k-2,1^{2}\right]}$ as a $\operatorname{GL}(n, \mathbf{Z})$-module for $n \geq k+2$. To show this, it suffices to show that $\operatorname{Coker}\left(\left(\tau_{k, N}^{\prime}\right)_{\mathbf{Q}}\right)$ is generated by

$$
\left(\begin{array}{c}
n+k-1 \\
k
\end{array}\right)+\frac{(k-2)(k-1)}{2}\left(\begin{array}{c}
n+k-3 \\
k
\end{array}\right)
$$

elements for $n \geq k+2$ since we have already shown that $\operatorname{Coker}\left(\left(\tau_{k, N}^{\prime}\right)_{\mathbf{Q}}\right) \supset S^{k} H_{\mathbf{Q}} \oplus$ $H_{\mathbf{Q}}^{\left[k-2,1^{2}\right]}$.

In general, $H^{*} \otimes \mathbf{z} \mathcal{L}_{n}^{N}(k+1)$ is generated by

$$
\mathfrak{G}:=\left\{x_{i}^{*} \otimes\left[x_{i_{1}}, x_{i_{2}}, \ldots, x_{i_{k+1}}\right] \mid 1 \leq i, i_{j} \leq n\right\} .
$$

Hence $\operatorname{Coker}\left(\tau_{k, N}^{\prime}\right)$ is also generated by these elements.

Lemma 5.7. For $n \geq 3$ and $k \geq 1$,

$$
x_{i}^{*} \otimes\left[x_{i_{1}}, x_{i_{2}}, \ldots, x_{i_{k+1}}\right]=0 \in \operatorname{Coker}\left(\tau_{k, N}^{\prime}\right)
$$

if $i_{l} \neq i$ for $1 \leq l \leq k+1$.

Proof. We show the lemma by induction on $k$. For $k=1$, we have $\tau_{1, N}^{\prime}\left(K_{i i_{1} i_{2}}\right)=$ $x_{i}^{*} \otimes\left[x_{i_{1}}, x_{i_{2}}\right]$. Assume $k \geq 2$. By the inductive hypothesis, there exists a certain $\sigma \in \mathcal{A}_{n}^{\prime}(k-1)$ such that

$$
\tau_{k-1, N}^{\prime}(\sigma)=x_{i}^{*} \otimes\left[x_{i_{1}}, x_{i_{2}}, \ldots, x_{i_{k}}\right] .
$$

On the other hand, we have $\tau_{1, N}^{\prime}\left(K_{i i_{k+1}}\right)=x_{i}^{*} \otimes\left[x_{i}, x_{i_{k+1}}\right]$. Then

$$
\tau_{k, N}^{\prime}\left(\left[K_{i i_{k+1}}, \sigma\right]\right)=x_{i}^{*} \otimes\left[x_{i_{1}}, x_{i_{2}}, \ldots, x_{i_{k+1}}\right] .
$$

This completes the proof of Lemma 5.7

Let $\mathfrak{F}$ be a set consisting of elements $x_{i}^{*} \otimes\left[x_{i_{1}}, x_{i_{2}}, \ldots, x_{i_{k+1}}\right]$ of $\mathfrak{G}$ such that $i_{l}=i$ for some $1 \leq l \leq n$, and $i_{m} \neq i$ for $m \neq l$.

Lemma 5.8. For $n \geq k+1$, $\operatorname{Coker}\left(\tau_{k, N}^{\prime}\right)$ is generated by $\mathfrak{F}$. 
Proof. Take any $x_{i}^{*} \otimes\left[x_{i_{1}}, x_{i_{2}}, \ldots, x_{i_{k+1}}\right] \in \mathfrak{G}$ such that $i_{l_{1}}=i_{l_{2}}=i$ for distinct $l_{1}, l_{2}$. Since $n \geq k+1$, there exists a certain $j \in\{1,2, \ldots, n\}$ such that $j \neq i, i_{l}$ for $1 \leq l \leq k+1$. Set

Then

$$
\sigma:= \begin{cases}K_{i j i_{k+1},}, & i \neq i_{k+1}, \\ K_{i j}^{-1}, & i=i_{k+1} .\end{cases}
$$

$$
\tau_{1, N}^{\prime}(\sigma)=x_{i}^{*} \otimes\left[x_{j}, x_{i_{k+1}}\right] .
$$

On the other hand, from Lemma 5.7, there exists a certain $\sigma^{\prime} \in \mathcal{A}_{n}^{\prime}(k-1)$ such that

Then we obtain

$$
\tau_{k-1, N}^{\prime}\left(\sigma^{\prime}\right)=x_{j}^{*} \otimes\left[x_{i_{1}}, x_{i_{2}}, \ldots, x_{i_{k}}\right]
$$

$$
\begin{aligned}
\tau_{k, N}^{\prime}\left(\left[\sigma, \sigma^{\prime}\right]\right)=x_{i}^{*} \otimes & {\left[x_{i_{1}}, x_{i_{2}}, \ldots, x_{i_{k+1}}\right] } \\
& -\sum_{l=1}^{k} \delta_{i i_{l}} x_{j}^{*} \otimes\left[x_{i_{1}}, \ldots, x_{i_{l-1}},\left[x_{j}, x_{i_{k+1}}\right], x_{i_{l+1}}, \ldots, x_{k}\right] .
\end{aligned}
$$

Observing the Jacobi identity

$$
[Z,[X, Y]]=[[Z, X], Y]-[[Z, Y], X]
$$

in the graded Lie algebra $\operatorname{gr}\left(\mathcal{A}_{n}^{\prime}\right)$, we see that the right-hand side of the equation above is equal to

$$
\begin{aligned}
& x_{i}^{*} \otimes\left[x_{i_{1}}, x_{i_{2}}, \ldots, x_{i_{k+1}}\right]+\delta_{i i_{1}} x_{j}^{*} \otimes\left[x_{j}, x_{i_{k+1}}, x_{i_{2}}, \ldots, x_{i_{k}}\right] \\
&-\sum_{l=2}^{k} \delta_{i i_{l}}\left(x_{j}^{*} \otimes\left[x_{i_{1}}, \ldots, x_{i_{l-1}}, x_{j}, x_{i_{k+1}}, x_{i_{l+1}}, \ldots, x_{k}\right]\right. \\
&\left.\quad-x_{j}^{*} \otimes\left[x_{i_{1}}, \ldots, x_{i_{l-1}}, x_{i_{k+1}}, x_{j}, x_{i_{l+1}}, \ldots, x_{k}\right]\right) .
\end{aligned}
$$

This completes the proof of Lemma 5.8

Lemma 5.9. For $n \geq 3$ and $k \geq 2$,

$$
x_{i}^{*} \otimes\left[x_{i_{1}}, x_{i_{2}}, \ldots, x_{i_{k}}, x_{i}\right]=0 \in \operatorname{Coker}\left(\tau_{k, N}^{\prime}\right)
$$

if $i_{l} \neq i$ for $1 \leq l \leq k$.

Proof. We show the lemma by induction on $k$. For $k=2$, we have

$$
\tau_{2, N}^{\prime}\left(\left[K_{i i_{1}}, K_{i i_{2}}\right]\right)=x_{i}^{*} \otimes\left[x_{i}, x_{i_{2}}, x_{i_{1}}\right]-x_{i}^{*} \otimes\left[x_{i}, x_{i_{1}}, x_{i_{2}}\right]=x_{i}^{*} \otimes\left[x_{i_{1}}, x_{i_{2}}, x_{i}\right] .
$$

Assume $k \geq 3$. By the inductive hypothesis, there exists a certain $\sigma \in \mathcal{A}_{n}^{\prime}(k-1)$ such that

$$
\tau_{k-1, N}^{\prime}(\sigma)=x_{i}^{*} \otimes\left[x_{i_{1}}, x_{i_{2}}, \ldots, x_{i_{k-1}}, x_{i}\right] .
$$

On the other hand, we have $\tau_{1, N}^{\prime}\left(K_{i i_{k}}\right)=x_{i}^{*} \otimes\left[i, i_{k}\right]$. Then, by the Jacobi identity,

$$
\begin{aligned}
\tau_{k, N}^{\prime}\left(\left[K_{i i_{k}}, \sigma\right]\right) & =x_{i}^{*} \otimes\left[x_{i_{1}}, x_{i_{2}}, \ldots, x_{i_{k-1}}, x_{i}, x_{i_{k}}\right]-x_{i}^{*} \otimes\left[x_{i_{1}}, x_{i_{2}}, \ldots, x_{i_{k-1}},\left[x_{i}, x_{i_{k}}\right]\right] \\
& =x_{i}^{*} \otimes\left[x_{i_{1}}, x_{i_{2}}, \ldots, x_{i_{k}}, x_{i}\right] .
\end{aligned}
$$

This completes the proof of Lemma 5.9.

Lemma 5.10. For $k \geq 5, n \geq k+2$ and $4 \leq l \leq k-1$,

$$
x_{i}^{*} \otimes\left[x_{i_{1}}, x_{i_{2}}, \ldots, x_{i_{l-1}}, x_{i}, x_{i_{l+1}}, \ldots, x_{i_{k+1}}\right]=0 \in \operatorname{Coker}\left(\tau_{k, N}^{\prime}\right)
$$

if $i_{m} \neq i$ for $m \neq l$. 
Proof. Since $n \geq k+2$, there exists a certain $j \in\{1,2, \ldots, n\}$ such that $j \neq i, i_{m}$ for $1 \leq m \leq k+1$ and $m \neq l$. From Lemma 5.7, there exist $\sigma \in \mathcal{A}_{n}^{\prime}(k-l+1)$ and $\tau \in \mathcal{A}_{n}^{\prime}(l-1)$ such that

$$
\begin{aligned}
\tau_{k-l+1, N}^{\prime}(\sigma) & =x_{i}^{*} \otimes\left[x_{j}, x_{i_{l+1}}, \ldots, x_{i_{k+1}}\right], \\
\tau_{l-1, N}^{\prime}(\tau) & =x_{j}^{*} \otimes\left[x_{i_{1}}, \ldots, x_{i_{l-1}}, x_{i}\right] .
\end{aligned}
$$

Then we have

$$
\tau_{k, N}^{\prime}([\sigma, \tau])=x_{i}^{*} \otimes\left[x_{i_{1}}, x_{i_{2}}, \ldots, x_{i_{l-1}}, x_{i}, x_{i_{l+1}}, \ldots, x_{i_{k+1}}\right] .
$$

This completes the proof of Lemma 5.10 .

Lemma 5.11. For $k \geq 2$,

$$
x_{i}^{*} \otimes\left[x_{i}, x_{i_{2}}, \ldots, x_{i_{k+1}}\right]=x_{j}^{*} \otimes\left[x_{j}, x_{i_{k+1}}, x_{i_{2}}, \ldots, x_{i_{k}}\right] \in \operatorname{Coker}\left(\tau_{k, N}^{\prime}\right)
$$

if $i, j \neq i_{2}, \ldots, i_{k+1}$ and $i \neq j$.

Proof. From Lemma 5.7, there exists a certain $\sigma \in \mathcal{A}_{n}^{\prime}(k-1)$ such that

$$
\tau_{k-1, N}^{\prime}(\sigma)=x_{j}^{*} \otimes\left[x_{i}, x_{i_{2}}, \ldots, x_{i_{k}}\right] .
$$

Then,

$$
\tau_{k, N}^{\prime}\left(\left[K_{i j i_{k+1}}, \sigma\right]\right)=x_{i}^{*} \otimes\left[x_{i}, x_{i_{2}}, \ldots, x_{i_{k+1}}\right]-x_{j}^{*} \otimes\left[x_{j}, x_{i_{k+1}}, x_{i_{2}}, \ldots, x_{i_{k}}\right] .
$$

This completes the proof of Lemma 5.11 .

Lemma 5.12. For $n \geq k+2$,

$$
x_{i}^{*} \otimes\left[x_{i}, x_{i_{2}}, \ldots, x_{i_{k+1}}\right]=x_{i}^{*} \otimes\left[x_{i}, x_{i_{3}}, \ldots, x_{i_{k+1}}, x_{i_{2}}\right] \in \operatorname{Coker}\left(\tau_{k, N}^{\prime}\right)
$$

if $i \neq i_{2}, \ldots, i_{k+1}$.

Proof. Since $n \geq k+2$, there exists a certain $j \in\{1,2, \ldots, n\}$ such that $j \neq i, i_{l}$ for $3 \leq l \leq k+1$. From Lemma [5.11, there exists a certain $\sigma \in \mathcal{A}_{n}^{\prime}(k-1)$ such that

$$
\tau_{k-1, N}^{\prime}(\sigma)=x_{i}^{*} \otimes\left[x_{i}, x_{i_{3}}, \ldots, x_{i_{k+1}}\right]-x_{j}^{*} \otimes\left[x_{j}, x_{i_{k+1}}, x_{i_{3}}, \ldots, x_{i_{k}}\right] .
$$

Then,

$$
\begin{gathered}
\tau_{k, N}^{\prime}\left(\left[\sigma, K_{i i_{2}}\right]\right)=x_{i}^{*} \otimes\left[x_{i}, x_{i_{2}}, \ldots, x_{i_{k+1}}\right]-x_{i}^{*} \otimes\left[x_{i}, x_{i_{3}}, \ldots, x_{i_{k+1}}, x_{i_{2}}\right] \\
-\delta_{j i_{2}} x_{i}^{*} \otimes\left[x_{j}, x_{i_{k+1}}, x_{i_{3}}, \ldots, x_{i_{k}}, x_{i}\right] .
\end{gathered}
$$

Hence from Lemma 5.9, we obtain the required result. This completes the proof of Lemma 5.12

Next, we consider the case where $k=3$.

Lemma 5.13. For $n \geq 4$, if $i \neq i_{1}, i_{2}, i_{4}$, then

(1) $x_{i}^{*} \otimes\left[x_{i_{1}}, x_{i_{2}}, x_{i}, x_{i_{4}}\right]=x_{i}^{*} \otimes\left[x_{i}, x_{i_{4}}, x_{i_{2}}, x_{i_{1}}\right]-x_{i}^{*} \otimes\left[x_{i}, x_{i_{4}}, x_{i_{1}}, x_{i_{2}}\right]$,

(2) $x_{i}^{*} \otimes\left[x_{i_{1}}, x_{i_{2}}, x_{i}, x_{i_{4}}\right]=x_{i}^{*} \otimes\left[x_{i_{2}}, x_{i_{4}}, x_{i}, x_{i_{1}}\right]$

in $\operatorname{Coker}\left(\tau_{3, N}^{\prime}\right)$.

Proof. From Lemma [5.9, there exists a certain $\sigma \in \mathcal{A}_{n}^{\prime}(2)$ such that

$$
\tau_{2}^{\prime}(\sigma)=x_{i}^{*} \otimes\left[x_{i_{1}}, x_{i_{2}}, x_{i}\right] .
$$


Then, we obtain

$$
\begin{aligned}
& \tau_{3, N}^{\prime}\left(\left[K_{i i_{4}}, \sigma\right]\right)=x_{i}^{*} \otimes\left[x_{i_{1}}, x_{i_{2}}, x_{i}, x_{i_{4}}\right]-x_{i}^{*} \otimes\left[x_{i_{1}}, x_{i_{2}},\left[x_{i}, x_{i_{4}}\right]\right] \\
& \quad=x_{i}^{*} \otimes\left[x_{i_{1}}, x_{i_{2}}, x_{i}, x_{i_{4}}\right]+x_{i}^{*} \otimes\left[x_{i}, x_{i_{4}},\left[x_{i_{1}}, x_{i_{2}}\right]\right] \\
& \quad=x_{i}^{*} \otimes\left[x_{i_{1}}, x_{i_{2}}, x_{i}, x_{i_{4}}\right]-x_{i}^{*} \otimes\left[x_{i}, x_{i_{4}}, x_{i_{2}}, x_{i_{1}}\right]+x_{i}^{*} \otimes\left[x_{i}, x_{i_{4}}, x_{i_{1}}, x_{i_{2}}\right] .
\end{aligned}
$$

Hence we have part (1). For part (2), from part (1), we have

$$
\begin{aligned}
& x_{i}^{*} \otimes\left[x_{i_{1}}, x_{i_{2}}, x_{i}, x_{i_{4}}\right]=x_{i}^{*} \otimes\left[x_{i}, x_{i_{4}}, x_{i_{2}}, x_{i_{1}}\right]-x_{i}^{*} \otimes\left[x_{i}, x_{i_{4}}, x_{i_{1}}, x_{i_{2}}\right], \\
& x_{i}^{*} \otimes\left[x_{i_{2}}, x_{i_{4}}, x_{i}, x_{i_{1}}\right]=x_{i}^{*} \otimes\left[x_{i}, x_{i_{1}}, x_{i_{4}}, x_{i_{2}}\right]-x_{i}^{*} \otimes\left[x_{i}, x_{i_{1}}, x_{i_{2}}, x_{i_{4}}\right]
\end{aligned}
$$

in $\operatorname{Coker}\left(\tau_{3, N}^{\prime}\right)$. Then from Lemma 5.12, we obtain the required result. This completes the proof of Lemma 5.13 .

Lemma 5.14. For $k \geq 5$ and $n \geq k+2$,

$$
x_{i}^{*} \otimes\left[x_{i_{1}}, x_{i_{2}}, \ldots, x_{i_{k-1}}, x_{i}, x_{i_{k+1}}\right]=0 \in \operatorname{Coker}\left(\tau_{k, N}^{\prime}\right)
$$

if $i_{l} \neq i$ for $l \neq k$.

Proof. Since $n \geq k+2$, there exists some $j \in\{1, \ldots, n\}$ such that $j \neq i_{l}, i$ for $1 \leq m \leq k+1$ and $m \neq k$. From Lemmas 5.13 and 5.7, there exist some $\sigma \in \mathcal{A}_{n}^{\prime}(3)$ and $\tau \in \mathcal{A}_{n}(k-3)$ such that

$$
\begin{aligned}
\tau_{3, N}^{\prime}(\sigma) & =x_{i}^{*} \otimes\left[x_{j}, x_{i_{k-1}}, x_{i}, x_{i_{k+1}}\right]-x_{i}^{*} \otimes\left[x_{i_{k-1}}, x_{i_{k+1}}, x_{i}, x_{j}\right], \\
\tau_{k-3, N}^{\prime}(\tau) & =x_{j}^{*} \otimes\left[x_{i_{1}}, \ldots, x_{i_{k-2}}\right],
\end{aligned}
$$

respectively. Then,

$$
\tau_{k, N}^{\prime}([\sigma, \tau])=x_{i}^{*} \otimes\left[x_{i_{1}}, x_{i_{2}}, \ldots, x_{i_{k-1}}, x_{i}, x_{i_{k+1}}\right] .
$$

This completes the proof of Lemma 5.14.

Lemma 5.15. For $k \geq 2$,

$$
\begin{aligned}
& x_{i}^{*} \otimes\left[x_{i_{1}}, x_{i_{2}}, x_{i}, x_{i_{4}}, \ldots, x_{i_{k+1}}\right] \\
& \quad=x_{j}^{*} \otimes\left[x_{j}, x_{i_{4}}, \ldots, x_{i_{k+1}}, x_{i_{2}}, x_{i_{1}}\right]-x_{j}^{*} \otimes\left[x_{j}, x_{i_{4}}, \ldots, x_{i_{k+1}}, x_{i_{1}}, x_{i_{2}}\right]
\end{aligned}
$$

$\in \operatorname{Coker}\left(\tau_{k, N}^{\prime}\right)$ if $i, j \neq i_{l}$ for $l \neq 3$, and $i \neq j$.

Proof. From Lemmas 5.9 and [5.7, there exist some $\sigma \in \mathcal{A}_{n}^{\prime}(k-2)$ and $\tau \in \mathcal{A}_{n}(2)$ such that

$$
\begin{aligned}
\tau_{2, N}^{\prime}(\sigma) & =x_{i}^{*} \otimes\left[x_{j}, x_{i_{4}}, \ldots, x_{i_{k+1}}\right], \\
\tau_{k-2, N}^{\prime}(\tau) & =x_{j}^{*} \otimes\left[x_{i_{1}}, x_{i_{2}}, x_{i}\right],
\end{aligned}
$$

respectively. Then, by the Jacobi identity,

$$
\begin{aligned}
\tau_{k, N}([\sigma, \tau])= & x_{i}^{*} \otimes\left[x_{i_{1}}, x_{i_{2}}, x_{i}, x_{i_{4}}, \ldots, x_{i_{k+1}}\right] \\
& \quad-x_{j}^{*} \otimes\left[x_{i_{1}}, x_{i_{2}},\left[x_{j}, x_{i_{4}}, \ldots, x_{i_{k+1}}\right]\right] \\
= & x_{i}^{*} \otimes\left[x_{i_{1}}, x_{i_{2}}, x_{i}, x_{i_{4}}, \ldots, x_{i_{k+1}}\right] \\
& \quad+x_{j}^{*} \otimes\left[x_{j}, x_{i_{4}}, \ldots, x_{i_{k+1}}, x_{i_{1}}, x_{i_{2}}\right]-x_{j}^{*} \otimes\left[x_{j}, x_{i_{4}}, \ldots, x_{i_{k+1}}, x_{i_{2}}, x_{i_{1}}\right] .
\end{aligned}
$$

This completes the proof of Lemma 5.15.

Lemma 5.16. For $k \geq 5$, $x_{i}^{*} \otimes\left[x_{i_{1}}, x_{i_{2}}, x_{i}, x_{i_{4}}, \ldots, x_{i_{k+1}}\right]=x_{j}^{*} \otimes\left[x_{i_{1}}, x_{i_{2}}, x_{j}, x_{i_{5}}, \ldots, x_{i_{k+1}}, x_{i_{4}}\right] \in \operatorname{Coker}\left(\tau_{k, N}^{\prime}\right)$ if $i, j \neq i_{l}$ for $l \neq 3$, and $i \neq j$. 
Proof. From Lemma [5.7, there exists some $\sigma \in \mathcal{A}_{n}(k-1)$ such that

$$
\tau_{k-1, N}^{\prime}(\sigma)=x_{i}^{*} \otimes\left[x_{i_{1}}, x_{i_{2}}, x_{j}, x_{i_{5}}, \ldots, x_{i_{k+1}}\right] .
$$

Then,

$$
\begin{aligned}
\tau_{k, N}^{\prime}\left(\left[\sigma, K_{j i_{4}}\right]\right)= & x_{i}^{*} \otimes\left[x_{i_{1}}, x_{i_{2}},\left[x_{i_{4}}, x_{i}\right], x_{i_{5}}, \ldots, x_{i_{k+1}}\right] \\
& \quad+x_{j}^{*} \otimes\left[x_{i_{1}}, x_{i_{2}}, x_{j}, x_{i_{5}}, \ldots, x_{i_{k+1}}, x_{i_{4}}\right] \\
= & x_{i}^{*} \otimes\left[x_{i_{1}}, x_{i_{2}}, x_{i_{4}}, x_{i}, x_{i_{5}}, \ldots, x_{i_{k+1}}\right] \\
& \quad-x_{i}^{*} \otimes\left[x_{i_{1}}, x_{i_{2}}, x_{i}, x_{i_{4}}, x_{i_{5}}, \ldots, x_{i_{k+1}}\right] \\
& \quad+x_{j}^{*} \otimes\left[x_{i_{1}}, x_{i_{2}}, x_{j}, x_{i_{5}}, \ldots, x_{i_{k+1}}, x_{i_{4}}\right] .
\end{aligned}
$$

Hence from Lemma 5.10, we obtain the required result. This completes the proof of Lemma 5.16.

In the following, we consider the case where $n \geq k+2$. From Lemmas 5.9, 5.10 and 5.14 we see that $\operatorname{Coker}\left(\tau_{k, N}^{\prime}\right)$ is generated by elements $x_{i}^{*} \otimes\left[x_{i}, x_{i_{2}}, \ldots, x_{i_{k+1}}\right]$ and $x_{i}^{*} \otimes\left[x_{i_{1}}, x_{i_{2}}, x_{i}, x_{i_{4}}, \ldots, x_{i_{k+1}}\right]$ of $\mathfrak{F}$ such that $1 \leq i, i_{l} \leq n$ and $i \neq i_{l}$. Furthermore, if we set

$$
s^{\prime}\left(i, i_{2}, \ldots, i_{k+1}\right):=x_{i}^{*} \otimes\left[x_{i}, x_{i_{2}}, \ldots, x_{i_{k+1}}\right] \in \operatorname{Coker}\left(\tau_{k, N}^{\prime}\right)
$$

for $i_{l} \neq i$, then from Lemmas 5.11 and 5.12 , we see that $s^{\prime}\left(i, i_{2}, \ldots, i_{k+1}\right)$ does not depend on the choice of $i$ such that $i \neq i_{l}$ for $2 \leq l \leq k+1$. Hence we can set

$$
s\left(i_{2}, \ldots, i_{k+1}\right):=s^{\prime}\left(i, i_{2}, \ldots, i_{k+1}\right)
$$

and have

$$
s\left(i_{2}, \ldots, i_{k+1}\right)=s\left(i_{3}, \ldots, i_{k+1}, i_{2}\right)=\cdots=s\left(i_{k+1}, i_{2}, \ldots, i_{k}\right)
$$

in $\operatorname{Coker}\left(\tau_{k, N}^{\prime}\right)$.

Next, set

$$
u^{\prime}\left(i_{1}, i_{2}, i, i_{4}, \ldots, i_{k+1}\right):=x_{i}^{*} \otimes\left[x_{i_{1}}, x_{i_{2}}, x_{i}, x_{i_{4}}, \ldots, x_{i_{k+1}}\right] \in \operatorname{Coker}\left(\tau_{k, N}^{\prime}\right)
$$

for $i_{l} \neq i$. From Lemma 5.15, we verify that

$$
u^{\prime}\left(i_{1}, i_{2}, i, i_{4}, \ldots, i_{k+1}\right)=s\left(i_{4}, \ldots, i_{k+1}, i_{2}, i_{1}\right)-s\left(i_{4}, \ldots, i_{k+1}, i_{1}, i_{2}\right)
$$

and it also does not depend on the choice of $i$ such that $i \neq i_{l}$ for $l \neq 3$. Hence we can set

$$
u\left(i_{1}, i_{2}, i_{4}, \ldots, i_{k+1}\right):=u^{\prime}\left(i_{1}, i_{2}, i, i_{4}, \ldots, i_{k+1}\right) .
$$

Here we consider some relations among the $u\left(i_{1}, i_{2}, i_{4}, \ldots, i_{k+1}\right) \mathrm{s}$. First, using

$$
u\left(i_{1}, i_{2}, i_{4}, \ldots, i_{k+1}\right)=s\left(i_{1}, i_{4}, \ldots, i_{k+1}, i_{2}\right)-s\left(i_{1}, i_{2}, i_{4}, \ldots, i_{k+1}\right),
$$

we obtain

$$
u\left(j, j_{1}, j_{2}, \ldots, j_{k}\right)+u\left(j, j_{2}, \ldots, j_{k}, j_{1}\right)+\cdots+u\left(j, j_{k}, j_{1}, \ldots, j_{k-1}\right)=0 .
$$

From Lemma 5.16, we see

$$
u\left(i_{1}, i_{2}, i_{4}, \ldots, i_{k+1}\right)=u\left(i_{1}, i_{2}, i_{5}, \ldots, i_{k+1}, i_{4}\right) .
$$


In general, for $k \geq 5$,

$$
\begin{aligned}
0= & x_{i}^{*} \otimes\left[x_{i_{1}}, x_{i_{2}},\left[x_{i}, x_{i_{4}}\right],\left[x_{i_{5}}, x_{i_{6}}\right], x_{i_{7}}, \ldots, x_{i_{k+1}}\right] \\
= & x_{i}^{*} \otimes\left[x_{i_{1}}, x_{i_{2}}, x_{i}, x_{i_{4}},\left[x_{i_{5}}, x_{i_{6}}\right], x_{i_{7}}, \ldots, x_{i_{k+1}}\right] \\
& \quad-x_{i}^{*} \otimes\left[x_{i_{1}}, x_{i_{2}}, x_{i_{4}}, x_{i},\left[x_{i_{5}}, x_{i_{6}}\right], x_{i_{7}}, \ldots, x_{i_{k+1}}\right] \\
= & x_{i}^{*} \otimes\left[x_{i_{1}}, x_{i_{2}}, x_{i}, x_{i_{4}},\left[x_{i_{5}}, x_{i_{6}}\right], x_{i_{7}}, \ldots, x_{i_{k+1}}\right] \\
= & x_{i}^{*} \otimes\left[x_{i_{1}}, x_{i_{2}}, x_{i}, x_{i_{4}}, x_{i_{5}}, x_{i_{6}}, x_{i_{7}}, \ldots, x_{i_{k+1}}\right] \\
& \quad-x_{i}^{*} \otimes\left[x_{i_{1}}, x_{i_{2}}, x_{i}, x_{i_{4}}, x_{i_{6}}, x_{i_{5}}, x_{i_{7}}, \ldots, x_{i_{k+1}}\right]
\end{aligned}
$$

in $\operatorname{Coker}\left(\tau_{k, N}^{\prime}\right)$. This shows

$$
u\left(i_{1}, i_{2}, i_{4}, i_{5}, i_{6}, i_{7}, \ldots, i_{k+1}\right)=u\left(i_{1}, i_{2}, i_{4}, i_{6}, i_{5}, i_{7}, \ldots, i_{k+1}\right) .
$$

Observing the fact that for any $l$, the symmetric group $\mathfrak{S}_{l}$ of degree $l$ is generated by a cyclic permutation of length $l$ and a transposition, we verify that

$$
u\left(i_{1}, i_{2}, j_{1}, j_{2}, \ldots, j_{k-2}\right)=u\left(i_{1}, i_{2}, j_{\gamma(1)}, j_{\gamma(2)}, \ldots, j_{\gamma(k-2)}\right)
$$

for any $\gamma \in \mathfrak{S}_{k-2}$ by (9) and (10).

In order to reduce the generators more, we consider the rational case. By the same argument as above, we see that $\operatorname{Coker}\left(\left(\tau_{k, N}^{\prime}\right)_{\mathbf{Q}}\right)$ is also generated by the $s\left(i_{2}, \ldots, i_{k+1}\right) \mathrm{s}$ and $u\left(i_{1}, i_{2}, i_{4} \ldots, i_{k+1}\right) \mathrm{s}$ as a $\mathbf{Q}$-vector space for $n \geq k+2$. Denote by $W$ the subspace of Coker $\left(\left(\tau_{k, N}^{\prime}\right)_{\mathbf{Q}}\right)$ generated by elements $u\left(i_{1}, i_{2}, i_{3}, \ldots, i_{k}\right)$ for $i_{1}>i_{2}>i_{3} \leq i_{4} \leq \cdots \leq i_{k}$. Then we have

Lemma 5.17. For $k \geq 5, n \geq k+2$, and any $1 \leq j_{1}, \ldots, j_{k} \leq n$,

$$
u\left(j_{1}, j_{2}, j_{3}, \ldots, j_{k}\right) \in W .
$$

Proof. By (11), we may assume that $j_{1}>j_{2}$ and $j_{3} \leq \cdots \leq j_{k+1}$. Suppose $j_{2} \leq j_{3}$. If $j_{2}<j_{3}$, by (8), we obtain

$$
\begin{gathered}
u\left(j_{1}, j_{2}, j_{3}, \ldots, j_{k}\right)=-u\left(j_{1}, j_{3}, j_{2}, j_{4}, \ldots, j_{k}\right)-u\left(j_{1}, j_{4}, j_{2}, j_{3}, j_{5}, \ldots, j_{k}\right) \\
-\cdots-u\left(j_{1}, j_{k}, j_{2}, j_{3}, \ldots, j_{k-1}\right) \in W .
\end{gathered}
$$

If $j_{2}=j_{3}$, there exists some $l$ such that $3 \leq l \leq k$ and

$$
j_{2}=j_{3}=\cdots=j_{l}<j_{l+1} \leq \cdots \leq j_{k} .
$$

Then, by (8), we see

$$
\begin{array}{r}
(l-1) u\left(j_{1}, j_{2}, j_{3}, \ldots, j_{k}\right)=-u\left(j_{1}, j_{l+1}, j_{2}, \ldots, j_{l}, j_{l+1}, \ldots j_{k}\right) \\
-\cdots-u\left(j_{1}, j_{k}, j_{2}, \ldots, j_{k-1}\right) .
\end{array}
$$

Therefore, we obtain the required result. This completes the proof of Lemma 5.17.

Now, if we set $V:=\operatorname{Coker}\left(\left(\tau_{k, N}^{\prime}\right)_{\mathbf{Q}}\right) / W$, we have

$$
s\left(j_{1}, j_{2}, j_{3}, \ldots, j_{k}\right)=s\left(j_{2}, j_{1}, j_{3}, \ldots, j_{k}\right) \in V .
$$

This shows

$$
s\left(j_{1}, j_{2}, j_{3}, \ldots, j_{k}\right)=s\left(j_{\gamma(1)}, j_{\gamma(2)}, j_{\gamma(3)}, \ldots, j_{\gamma(k)}\right) \in V
$$


for any $\gamma \in \mathfrak{S}_{k}$. In particular, $V$ is generated by $s\left(j_{1}, j_{2}, j_{3}, \ldots, j_{k}\right)$ such that $1 \leq j_{1} \leq \cdots \leq j_{k} \leq n$. Therefore we conclude that

Proposition 5.1. For $k \geq 5$ and $n \geq k+2$, $\operatorname{Coker}\left(\left(\tau_{k, N}^{\prime}\right)_{\mathbf{Q}}\right)$ is generated by

$$
\left\{s\left(i_{1}, i_{2}, i_{3}, \ldots, i_{k}\right) \mid 1 \leq i_{1} \leq \cdots \leq i_{k} \leq n\right\}
$$

and

$$
\left\{u\left(i_{1}, i_{2}, i_{3}, \ldots, i_{k}\right) \mid i_{1}>i_{2}>i_{3} \leq i_{4} \leq \cdots \leq i_{k}\right\}
$$

as a $\mathbf{Q}$-vector space. In particular, the number of the generators above is

$$
\left(\begin{array}{c}
n+k-1 \\
k
\end{array}\right)+\frac{(k-2)(k-1)}{2}\left(\begin{array}{c}
n+k-3 \\
k
\end{array}\right) .
$$

Therefore we conclude that

Theorem 5.3. For $n \geq k+2$,

$$
\operatorname{Coker}\left(\left(\tau_{k, N}^{\prime}\right)_{\mathbf{Q}}\right)=S^{k} H_{\mathbf{Q}} \oplus H_{\mathbf{Q}}^{\left[k-2,1^{2}\right]} .
$$

\section{ACKNOWLEDGMENTS}

A part of this work was done when the author stayed at Aarhus University in Denmark in 2009. He would like to thank Aarhus University for its hospitality. He would also like to express his thanks to the referee for his/her careful reading of our original paper and for giving him helpful comments. This research was supported by the Global COE program at Kyoto University.

\section{REFERENCES}

[1] S. Andreadakis; On the automorphisms of free groups and free nilpotent groups, Proc. London Math. Soc. (3) 15 (1965), 239-268. MR0188307 (32:5746)

[2] K. T. Chen; Integration in free groups, Ann. of Math. (2) 54, no. 1 (1951), 147-162. MR0042414 (13:105c)

[3] F. Cohen and J. Pakianathan; On Automorphism Groups of Free Groups, and Their Nilpotent Quotients, preprint.

[4] F. Cohen and J. Pakianathan; On subgroups of the automorphism group of a free group and associated graded Lie algebras, preprint.

[5] B. Farb; Automorphisms of $F_{n}$ which act trivially on homology, in preparation.

[6] W. Fulton; Young Tableaux, London Mathematical Society Student Texts 35, Cambridge University Press (1997). MR1464693 (99f:05119)

[7] R. Hain, Infinitesimal presentations of the Torelli group, Journal of the American Mathematical Society 10 (1997), 597-651. MR1431828(97k:14024)

[8] P. Hall; A contribution to the theory of groups of prime-power order. Proc. London Math. Soc., II. s. 36 (1933), 29-95.

[9] M. Hall; The theory of groups, The Macmillan Co., New York, N.Y. (1959). MR0103215 $(21: 1996)$

[10] D. Johnson; An abelian quotient of the mapping class group, Math. Ann. 249 (1980), 225-242. MR.579103 (82a:57008)

[11] D. Johnson; The structure of the Torelli group III: The abelianization of $\mathcal{I}_{g}$, Topology 24 (1985), 127-144. MR793179 (87a:57016)

[12] N. Kawazumi; Cohomological aspects of Magnus expansions, preprint, arXiv: math.GT/0505497.

[13] S. Krstić, J. McCool; The non-finite presentability in $I A\left(F_{3}\right)$ and $G L_{2}\left(\mathbf{Z}\left[t, t^{-1}\right]\right)$, Invent. Math. 129 (1997), 595-606. MR1465336 (98h:20053)

[14] W. Magnus; Über $n$-dimensionale Gittertransformationen, Acta Math. 64 (1935), 353-367. MR.1555401

[15] W. Magnus, A. Karras, D. Solitar; Combinatorial group theory, Interscience Publ., New York (1966). MR2109550 (2005h:20052) 
[16] J. McCool; Some remarks on IA automorphisms of free groups, Can. J. Math. Vol. XL, no. 5 (1998), 1144-1155. MR973513 (90d:20057)

[17] S. Morita; Families of Jacobian manifolds and characteristic classes of surface bundles I, Ann. Inst. Fourier 39 (1989), 777-810. MR.1030850 (91d:32028)

[18] S. Morita; Abelian quotients of subgroups of the mapping class group of surfaces, Duke Mathematical Journal 70 (1993), 699-726. MR1224104 (94d:57003)

[19] S. Morita; Structure of the mapping class groups of surfaces: A survey and a prospect, Geometry and Topology Monographs Vol. 2 (1999), 349-406. MR 1734418 (2000j:57039)

[20] S. Morita; Cohomological structure of the mapping class group and beyond, Proc. of Symposia in Pure Math. 74 (2006), 329-354. MR2264550(2007j:20079)

[21] J. Nielsen; Die Isomorphismen der allgemeinen unendlichen Gruppe mit zwei Erzeugenden, Math. Ann. 78 (1918), 385-397. MR1511907

[22] J. Nielsen; Die Isomorphismengruppe der freien Gruppen, Math. Ann. 91 (1924), 169-209. MR 1512188

[23] J. Nielsen; Untersuchungen zur Topologie der geschlossenen Zweiseitigen Fläschen, Acta Math. 50 (1927), 189-358. MR.1555256

[24] A. Pettet; The Johnson homomorphism and the second cohomology of $I A_{n}$, Algebraic and Geometric Topology 5 (2005), 725-740. MR2153110 (2006j:20050)

[25] C. Reutenauer; Free Lie Algebras, London Mathematical Society Monographs, new series, no. 7, Oxford University Press (1993). MR.1231799 (94j:17002)

[26] T. Satoh; New obstructions for the surjectivity of the Johnson homomorphism of the automorphism group of a free group, Journal of the London Mathematical Society, (2) 74 (2006), 341-360. MR2269583 (2007i:20060)

[27] T. Satoh; The cokernel of the Johnson homomorphisms of the automorphism group of a free metabelian group, Transactions of American Mathematical Society, 361 (2009), 2085-2107. MR2465830 (2009i:20073)

[28] T. Satoh; On the fourth Johnson homomorphism of the automorphism group of a free group, preprint.

[29] E. Witt; Treue Darstellung Liescher Ringe, Journal für die Reine und Angewandte Mathematik, 177 (1937), 152-160.

[30] V. M. Zhuravlev; A free Lie algebra as a module over the full linear group, Sbornik Mathematics 187 (1996), 215-236. MR1392842 (97f:20053)

Department of Mathematics, Graduate School of Science, Kyoto University, KiTASHIRAKaWAOIWAKe CHO, SAKYO-KU, KYOTO City 606-8502, JAPAN

E-mail address: takao@math.kyoto-u.ac.jp 